\title{
Development and Characterization of PLGA Nanoparticle-Laden Hydrogels for Sustained Ocular Delivery of Norfloxacin in the Treatment of Pseudomonas Keratitis: An Experimental Study
}

This article was published in the following Dove Press journal:

Drug Design, Development and Therapy

\author{
Rana M Gebreel' \\ Noha A Edris ${ }^{2}$ \\ Hala M Elmofty ${ }^{2}$ \\ Mina I Tadros (D) 3,4 \\ Mohamed A El-Nabarawi ${ }^{3}$ \\ Doaa H Hassan (iD' \\ 'Department of Pharmaceutics, College \\ of Pharmaceutical Sciences and Drug \\ Manufacturing, Misr University for \\ Science and Technology, Giza, Egypt; \\ ${ }^{2}$ Department of Ophthalmology, Faculty \\ of Medicine, Cairo University, Cairo, \\ Egypt; ${ }^{3}$ Department of Pharmaceutics \\ and Industrial Pharmacy, Faculty of \\ Pharmacy, Cairo University, Cairo, Egypt; \\ ${ }^{4}$ Department of Pharmaceutics, Faculty of \\ Pharmacy and Drug Technology, Egyptian \\ Chinese University, Cairo, Egypt
}

\begin{abstract}
Aim: Norfloxacin (NFX) has low ocular bioavailability. The current work aimed to develop NFX-loaded nanoparticle (NP)-laden hydrogels to improve the ocular potential of NFX, minimize the need for frequent instillations and lower undesirable side effects.

Methods: NFX-loaded NPs were developed via the double-emulsion/solvent evaporation technique, according to $2^{1} .4^{1}$ full factorial design, using two types of polylactic-co-glycolic acid (PLGA) polymer and four (drug: polymer) ratios. NPs were evaluated for particle size (PS), polydispersity index (PDI), zeta potential (ZP), drug entrapment efficiency percentage (EE\%), drug percentage released after $30 \mathrm{~min}\left(\mathrm{Q}_{30 \mathrm{~min}}\right)$ and 12 hours $\left(\mathrm{Q}_{12 \mathrm{~h}}\right)$, drug percentage permeated through goat corneas after $30 \mathrm{~min}\left(\mathrm{P}_{30 \mathrm{~min}}\right)$ and 12 hours $\left(\mathrm{P}_{12 \mathrm{~h}}\right)$ and morphology. Two formulae were statistically selected and incorporated into hydroxypropyl methylcellulose (HPMC)-based hydrogels; G1 - G4. The latter systems were evaluated for appearance, clarity, $\mathrm{pH}$, spreadability, rheology, drug percentages released, drug percentages permeated, antimicrobial activity against Pseudomonas aeruginosa, and histopathological changes.

Results: The selected NPs (NP2 and NP6) were spherical in shape and possessed suitable PS (392.02 $\mathrm{nm}$ and $190.51 \mathrm{~nm})$ and PDI (0.17 and 0.18), high magnitude of ZP ( $-30.43 \mathrm{mV}$ and $-33.62 \mathrm{mV}$ ), high EE\% (79.24\% and 91.72\%), low $\mathrm{Q}_{30 \min }(10.96 \%$ and $16.65 \%)$ and $\mathrm{P}_{30 \mathrm{~min}}$ (17.39\% and 21.05\%) and promising $\mathrm{Q}_{12 \mathrm{~h}}(58.23 \%$ and $71.20 \%)$ and $\mathrm{P}_{12 \mathrm{~h}}(53.31 \%$ and $65.01 \%$ ), respectively. Clear, spreadable, tolerable, pseudoplastic, and thixotropic HPMCbased hydrogels were developed. They showed more prolonged drug release and drug permeation profiles. NP2- and NP6-laden hydrogels (G3 and G4 systems, respectively) had promising antibacterial activity, and reasonable histopathological safety.
\end{abstract}

Conclusion: G3 and G4 are potential ocular delivery systems for NFX.

Keywords: norfloxacin, nanoparticles, PLGA, ocular delivery, Pseudomonas keratitis

\section{Introduction}

Poor ophthalmic bioavailability of drugs $(<1 \%)$ from common eye drops occurs mostly due to the precorneal loss factors like the quick tear turnover, the nonproductive drug absorption, the momentary residence time in the cul-de-sac, and the poor drug permeability to the corneal epithelial membrane. ${ }^{1,2}$ The limited ocular bioavailability of drugs favors the need for frequent instillation to accomplish the targeted therapeutic effect, which may lead to undesirable side effects as a result of systemic drug absorption. The efficiency of an ophthalmic drug delivery system
Correspondence: Mina I Tadros

Tel $+20 \quad 1223620458$

Fax +20223628246

Email mina.tadros@pharma.cu.edu.eg 
depends on the drug bioavailability, which could be enhanced by extending the pre-corneal drug residence time and promoting corneal drug penetration. ${ }^{3}$

Keratitis is an ocular disease that can result from microbial infection (eg, bacteria, fungi, viruses, or protozoans) or from non-infectious damage, such as that caused by eye trauma or exposure to chemicals or ultraviolet light. Microbial keratitis is considered a sight-threatening infection that can cause ulceration of the cornea and produce scarring, neovascularization, and loss of vision in severe cases. ${ }^{4}$ Poor patient compliance is usually encountered with microbial keratitis since a frequent instillation schedule every 2-4 hours is needed for common infections and every $30 \mathrm{~min}$ for extreme corneal ulcers. Upon ocular instillation, the drug solution or suspension mixes with the lacrimal fluid and remains in contact with the mucosa for 1-2 minutes only due to the continuous release of lacrimal fluid. Furthermore, the drainage through the lower and the upper canaliculus into the lacrimal sac and then to the nasolacrimal duct would induce a rapid drug loss during blinking. ${ }^{5,6}$ The ocular drug bioavailability could be enhanced by altering the physicochemical characteristics of the drug and/or the development of specific nano-delivery systems.

Bacterial keratitis is commonly caused by Pseudomonas aeruginosa, rod-shaped, Gram-negative bacterium, especially among contact lens wearers. This rapidly progressive disease and can end in permanent loss of vision, if not treated appropriately. ${ }^{7}$ Fluoroquinolones supplanted other antibiotics as gold standards for treating bacterial keratitis for many reasons including, the broad-spectrum bactericidal activities, the better intraocular drug concentrations as well as the lower side effects and drug resistance potential. ${ }^{8}$ They act by targeting the bacterial enzymes required for DNA synthesis including, replication, transcription, repair, and recombination. ${ }^{9}$ Norfloxacin (NFX) is a synthetic fluoroquinolone antibiotic that is active against both Grampositive and Gram-negative bacteria. ${ }^{10}$ Unfortunately, NFX suffers from poor ocular bioavailability, possibly due to its limited aqueous solubility and the extensive precorneal drug drainage. ${ }^{11,12}$ For keratitis, it should be instilled $(0.3 \% ; 1-2$ drops) four times daily, up to seven days to maintain an effective therapeutic drug concentration.

Nanoparticles (NPs) represent promising ocular drug carriers since most of them can be simply developed under mild conditions to incorporate various bioactive compounds. Compared to the traditional eye preparations, NPs show remarkably enhanced drug absorption characteristics and slower drug clearance rates. In a parallel line, they are better tolerated by patients than larger microparticles. $^{13}$

Poly(dl-lactide-coglycolide) (PLGA) represent a wide series of biodegradable and biocompatible copolymers that possess either free carboxylic acid- or ester-terminated (lauryl- or methyl ester-) end groups. Their characteristics are affected by the lactic acid/glycolic acid ratio, stereochemistry of lactic acid (D, L, or DL), degree of crystallinity, and molecular weight. ${ }^{14}$ The development of relatively uniform and easily redispersed PLGA NPs necessitates the incorporation of a stabilizer like polyvinyl alcohol (PVA). ${ }^{15}$

Parallel to other mucoadhesive NPs, PLGA NPs are commonly used to achieve promising sustained drug delivery systems due to their favorable characteristics including, prolonged degradation kinetics, ease metabolism of PLGA monomers through the Krebs cycle in the body, ${ }^{16}$ and their ability to bypass P-gp efflux and hence, enhance the corneal drug permeation. ${ }^{17}$ Furthermore, the desired drug release profiles and the subsequent dosage schedule could be easily tailored. PLGA copolymers, possessing a lactide: coglycolide ratio of 50:50, were investigated in this work due to their balanced hydrophilic and lipophilic characteristics and promising degradation rates. ${ }^{18}$ The encapsulation of antibacterial agents in PLGA NPs was investigated to enhance the therapeutic drug efficacy as well as to minimize the undesirable side effects and possible microbial drug resistance. This was attributed to the improved distribution characteristics, better cellular uptake, and greater efficacy against both extracellular and intracellular pathogens. ${ }^{19}$

Polysaccharides like pectin, chitosan, chitosan, guar gum, sodium alginate, and hydroxypropyl methylcellulose (HPMC) have been extensively investigated to develop ocular hydrogels, incorporating mucoadhesive NPs. A limited number of studies investigated the ocular potential of incorporating PLGA NPs in hydrogels like; alginate-chitosan in-situ gels, ${ }^{20}$ Poloxamer 407 thermosensitive gels, ${ }^{21}$ and Carbomer 934 mucoadhesive gels. $^{22}$ Herein, PLGA NP-laden HPMC hydrogels were developed in an attempt to merge the advantages of PLGA NPs and HPMC hydrogels and develop a more promising drug delivery system that can solve the disadvantages of the rapid drainage of the NPs aqueous dispersion, avoid the burst release of the surface-loaded drug, 
and prolong the precorneal drug residence time. The variables influencing the development of NFX-loaded PLGA NPs (NP1 - NP8), according to $2^{1} .4^{1}$ full factorial design, were investigated. Based on the statistical analysis of the dependent variables, the best achieved NPs were incorporated into HPMC-based hydrogels. The antimicrobial activity against Pseudomonas aeruginosa and the histopathological safety of the best-achieved systems were explored.

\section{Materials and Methods Materials}

Norfloxacin (NFX) was received as a kind gift from Eipico (Cairo, Egypt). Poly(lactic-co-glycolic acid) (PLGA; PURASORB $^{\circledR}$ PDLG 5002 (ester-terminated copolymer of DL-lactide: glycolide in a 50/50 molar ratio; inherent viscosity midpoint $=0.2 \mathrm{dL} / \mathrm{g}$ ) and PURASORB PDLG 5002A (carboxylic acid-terminated copolymer of DL-lactide: glycolide in a 50/50 molar ratio; inherent viscosity midpoint $=0.2 \mathrm{dL} / \mathrm{g}$ ) were purchased from Purac Biomaterial (Ingelheim, Germany). Polyvinyl alcohol (PVA; MW 95,000) was purchased from Sigma-Aldrich Chemie GmbH (Steinheim, Germany). Spectra Por@ semipermeable membrane tubing $(12-14 \mathrm{kDa})$ was derived from Spectrum Laboratories Inc, (Rancho Dominguez, CA). Hydroxypropyl methylcellulose K4M (HPMC) was purchased from Loba Chemie pvt. Ltd., (Mumbai, India). Sodium chloride, disodium hydrogen phosphate, potassium dihydrogen phosphate, and chloroform were purchased from El-Nasr Pharmaceutical Chemicals Co. (Cairo, Egypt.) All other chemicals were of analytical grade and used as received.

\section{Preparation of NFX-Loaded PLGA NPs}

NFX-loaded PLGA NPs were prepared by a modified double-emulsion/solvent evaporation technique. ${ }^{23}$ Solutions of NFX $(0.3 \%)$ and ester-terminated or acidterminated PLGA copolymers at four (drug: polymer) ratios $(1: 0.5,1: 1,1: 1.5$ or $1: 2)$ were prepared in chloroform, at room temperature, with magnetic stirring (Jenway 1200, Staffordshire, UK). A primary w/o emulsion was developed following the incorporation of PVA solution in deionized water $(1.5 \%, \mathrm{w} / \mathrm{v})$ into the organic phase with high-speed homogenization (Heidolph homogenizer DIAX 900, Kelheim, Germany). A w/o/w emulsion was developed following the addition of the primary emulsion, in a dropwise manner at a constant rate $(0.5 \mathrm{~mL} / \mathrm{min})$, into the continuous phase (PVA solution $1.5 \%$, w/v) with magnetic stirring. The emulsion was left on gentle stirring overnight to allow solvent evaporation. The NPs were separated by centrifugation at $15,000 \mathrm{rpm}$ for $30 \mathrm{~min}$ at $4{ }^{\circ} \mathrm{C}$ using a cooling centrifuge (Sigma 3-30 KS, Osterode am Harz, Germany) and washed with deionized water. The composition of the investigated formulae is shown in Table 1.

\section{Screening of the Formulation Variables Influencing the Design of NFX-Loaded PLGA NPs}

The formulation variables influencing the design of NFXloaded PLGA NPs were screened using $2^{1} .4^{1}$ full factorial design. Two independent variables were evaluated, polymer type (X1) at two levels and drug: polymer ratio (X2) at four levels. The dependent responses were particle size (PS, Y1), zeta potential magnitude (ZP, Y2), drug entrapment efficiency percentage (EE\%, Y3), drug percentage released after 30 min $\left(Q_{30 \min }, Y 4\right)$ and 12 hours $\left(Q_{12 h}\right.$, Y5), drug percentage permeated through goat corneas after 30 min $\left(\mathrm{P}_{30 \mathrm{~min}}, \mathrm{Y} 6\right)$ and 12 hours $\left(\mathrm{P}_{12 \mathrm{~h}}, \mathrm{Y} 7\right)$. The results were statistically analyzed using Design-Expert ${ }^{\circledR}$ software version 11 (Stat Ease, Inc., Minneapolis, MN, USA) to explore the significance of each variable via ANOVA.

\section{In vitro Characterization of NFX-Loaded PLGA NPs}

Particle Size and Zeta Potential

The particle size (PS), polydispersity index (PDI), and zeta potential (ZP) of NFX-loaded PLGA NPs were measured after appropriate dilution, in triplicates, via dynamic laser light scattering (Malvern Zetasizer Nano ZS Zen 3600, Worcestershire, England) at $25 \pm 0.5{ }^{\circ} \mathrm{C}$. The latter technique analyses the fluctuation of intensity of a scattered laser beam caused by the Brownian random motion of the particles, at an angle of $90^{\circ}$, as a function of time. ${ }^{24}$ The data were analyzed to determine the diffusion coefficient, which is needed to estimate the hydrodynamic diameter of NPs based on Stoke-Einstein equation. ${ }^{25}$

\section{Drug Entrapment Efficiency Percentage}

The drug entrapment efficiency percentage $(\mathrm{EE} \%)$ was measured by an indirect method. Briefly, the NP dispersion was centrifuged (Heraeus Megafuge 1.0 R; Hanau, Germany) at $10,000 \mathrm{rpm}$ for $15 \mathrm{~min}$. The amount of free NFX in the supernatant was measured 


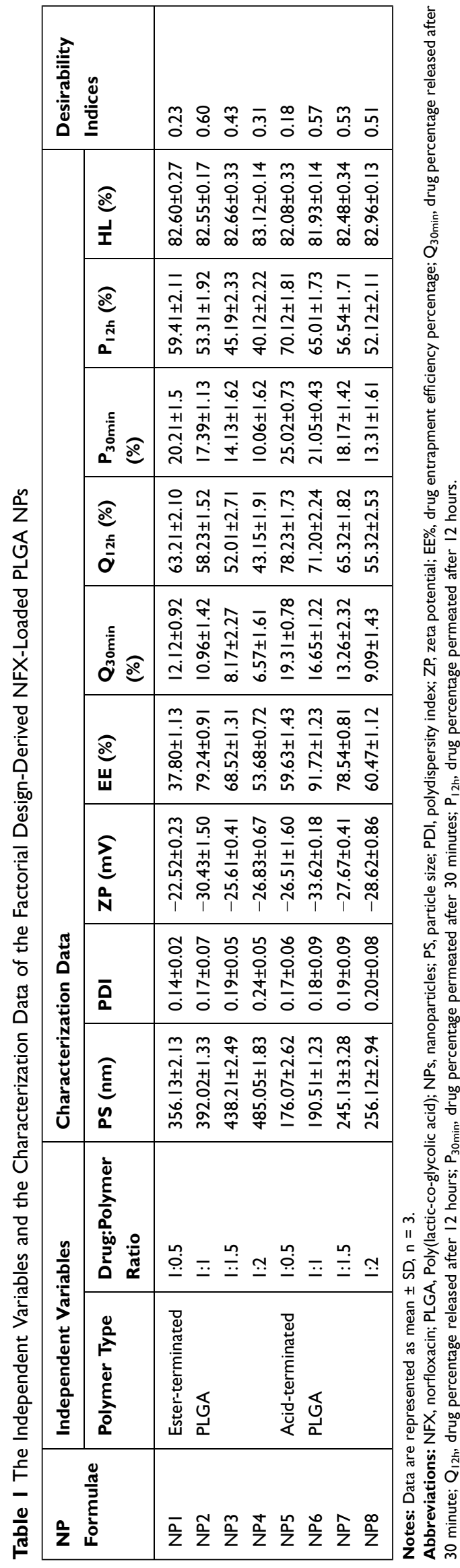

spectrophotometrically at a predetermined $\lambda_{\max }$ of $281 \mathrm{~nm}$ (Shimadzu UV 1650 UV-VIS spectrophotometer; Kyoto, Japan). EE\% was determined using equation (1)

$$
\mathrm{EE}(\%)=\frac{\begin{array}{c}
\text { Total loaded } \\
\text { drug amount }
\end{array}-\begin{array}{c}
\text { free drug } \\
\text { amount in } \\
\text { supernatant }
\end{array}}{\text { Total loaded drug amount }} \times 100
$$

\section{Drug Release Studies}

The in vitro release studies of NFX from NFX-loaded PLGA NPs were performed in a USP dissolution tester apparatus (type II) (Pharmatron DIS 6000, Thun, Switzerland). A sample of each drug-loaded (0.3\%) NPs dispersion was placed in a glass cylindrical tube that was tightly covered with a Spectra Por ${ }^{\mathcal{O}}$ semi-permeable membrane tubing from one end. The dispersion-loaded tube was attached with plastic zip ties to the paddle of the apparatus. The latter was adjusted to rotate $(25 \mathrm{rpm})$ in a volume of $50 \mathrm{~mL}$ of simulated tear fluid (STF, pH 7.4) at $34 \pm 0.5^{\circ} \mathrm{C}$ to simulate the ocular surface temperature. ${ }^{26}$ Aliquot samples $(3 \mathrm{~mL})$ were withdrawn up to 12 hours and were immediately replenished with equal volumes of fresh dissolution medium. The drug percentages released were determined spectrophotometrically at $\lambda_{\max } 281$ $\mathrm{nm}$, as previously noted. The studies were performed in triplicates and the mean ( \pm S.D.) values of the drug percentages released were plotted vs time. ${ }^{27}$ The drug percentages released after $30 \mathrm{~min}\left(\mathrm{Q}_{30 \mathrm{~min}}\right)$ and 12 hours $\left(\mathrm{Q}_{12 \mathrm{~h}}\right)$ were calculated. Parallel in vitro drug release studies of an aqueous NFX suspension $(0.3 \%)$ were conducted to investigate the potential of NPs.

\section{Ex vivo Characterization of NFX-Loaded PLGA NPs}

\section{Transcorneal Drug Permeation Studies}

Goat corneas are commonly used to study the transcorneal permeability of drugs. ${ }^{28,29}$ Within $30 \mathrm{~min}$ of the sacrifice of the animals, fresh whole eyeballs of goats were obtained from a local slaughterhouse, soaked in normal saline, and transported to the laboratory in a cold chain. The carefully preserved corneas and the surrounding scleral tissue $(5-6 \mathrm{~mm})$ were stored in freshly prepared STF; pH 7.4.

The ex vivo corneal drug permeation studies were carried out, in triplicates, as noted before in the in vitro drug release studies. Yet, the glass cylindrical tubes were tightly covered with the excised goat corneas (instead of the semi-permeable membrane tubing) in such a way that the epithelial surfaces were facing the NP dispersions. The 
drug percentages permeated after 30 min $\left(\mathrm{P}_{30 \mathrm{~min}}\right)$ and 12 hours $\left(\mathrm{P}_{12 \mathrm{~h}}\right)$ were calculated. Parallel ex vivo corneal drug permeation studies of drug-free NPs dispersion (control) and an aqueous NFX suspension $(0.3 \%)$ were conducted to investigate the permeation enhancing effect of NPs.

\section{Corneal Hydration Levels}

Corneal hydration levels (HL\%) were calculated by the gravimetric method. ${ }^{30}$ Following the ex vivo corneal drug permeation studies, the corneas were carefully removed, gently blotted with filter paper to remove any surface water and weighed $\left(\mathrm{W}_{\mathrm{w}}\right)$. The corneas were desiccated at $100{ }^{\circ} \mathrm{C}$ for 6 hours and reweighed to determine the dry corneal weight $\left(\mathrm{W}_{\mathrm{d}}\right)$. The corneal HL\% was defined using equation (2)

$$
\mathrm{HL} \%=\frac{1-\mathrm{W}_{\mathrm{d}}}{\mathrm{W}_{\mathrm{w}}} \times 100
$$

\section{Selection of the Best Achieved NFX-Loaded PLGA NPs}

The best-achieved formulae were elaborated based on the estimated desirability index (DI) values. A desirability index of 1 expresses a completely desirable formula, while a desirability index of 0 expresses a completely undesirable one. ${ }^{31}$ The optimum formulae were proposed to achieve a compromise with 7 dependent responses (minimize; $\mathrm{PS}, \mathrm{Q}_{30 \mathrm{~min}}$ and $\mathrm{P}_{30 \mathrm{~min}}$, and maximize; $\mathrm{ZP}$ magnitude, $E E \%, \mathrm{Q}_{12 \mathrm{~h}}$ and $\mathrm{P}_{12 \mathrm{~h}}$ ).

\section{In vitro Characterization of the Best}

\section{Achieved NFX-Loaded PLGA NPs}

\section{Morphology}

The topography of the NPs was scanned using a transmission electron microscope (Philips CM-10, Eindhoven, The Netherlands). Representative samples of NP2 and NP6 were stained (2\% phosphotungstic acid solution) to examine their uniformity of shape, size, and presence of aggregates. ${ }^{32}$ Digital Micrograph and Olympus soft imaging viewer software (Alexandra, Singapore) were used to capture and analyze images.

\section{Fourier-Transform Infrared Spectroscopy (FT-IR) Studies}

Representative samples of NP2 and NP6 were pre-frozen at $-40{ }^{\circ} \mathrm{C}$ for 2 hours, and then lyophilized at $-40{ }^{\circ} \mathrm{C}$ for 48 hours. FT-IR spectra of the drug (NFX), esterterminated or acid-terminated PLGA polymer, 1: 1 drug: polymer physical mixtures, and the lyophilized samples were recorded on FT-IR spectrophotometer (Genesis II Mattson, USA) between $4500 \mathrm{~cm}^{-1}$ and $500 \mathrm{~cm}^{-1}$ using the potassium bromide disc technique. ${ }^{33}$

\section{Preparation of NFX-Loaded PLGA NP-Laden Hydrogels}

The hydrogels were prepared by gradually dispersing the calculated amounts of HPMC K4M $(1.5$ or $3 \%$, w/w) in half of the needed amount of deionized water at $70^{\circ} \mathrm{C}$, whilst stirring. The dispersions were kept overnight in a refrigerator to get air bubbles-free clear hydrogels. The final hydrogel volumes were adjusted with deionized water following the incorporation of the lyophilized optimum formulae; NP2 or NP6 so that the final drug concentration is $0.3 \%$. The composition of the investigated hydrogels (G1 - G4) is shown in Table 2.

\section{Characterization of NFX-Loaded PLGA NP-Laden Hydrogels \\ Visual Appearance, Clarity, and $\mathrm{pH}$}

The appearance, color, homogeneity, and clarity of the gels were visually evaluated under light against white and black backgrounds. The $\mathrm{pH}$ values of the appropriately

Table 2 The Composition and Characterization Data of the Investigated NFX-Loaded PLGA NP-Laden Hydrogels

\begin{tabular}{|c|c|c|c|c|c|c|c|c|c|c|}
\hline \multirow[t]{2}{*}{ Hydrogels } & \multicolumn{2}{|c|}{ Composition } & \multicolumn{8}{|c|}{ Characterization Data } \\
\hline & NPs & $\begin{array}{l}\text { HPMC } \\
\text { Concentration } \\
(\%, w / w)\end{array}$ & pH & $\begin{array}{l}\text { Travelled } \\
\text { Distance } \\
(\mathrm{cm})\end{array}$ & $\begin{array}{l}\eta_{\min } \\
(c p)\end{array}$ & $\begin{array}{l}\eta_{\max } \\
\text { (cp) }\end{array}$ & $\begin{array}{l}\text { Hysteresis } \\
\text { Loop Area } \\
\left(\mathrm{cm}^{2}\right)\end{array}$ & $\begin{array}{l}\text { Farrow's } \\
\text { Constant } \\
\text { (N) }\end{array}$ & $\begin{array}{l}\text { Flow } \\
\text { Behavior }\end{array}$ & $\begin{array}{l}\text { Zone of } \\
\text { Inhibition } \\
(\mathrm{mm})\end{array}$ \\
\hline GI & NP2 & 1.5 & $7.03 \pm 0.13$ & $7.16 \pm 0.30$ & $652 \pm 10$ & $1120 \pm 44$ & $21.03 \pm 1.14$ & 4.84 & Pseudoplastic & $27.0 \pm 0.5$ \\
\hline G2 & NP6 & & $6.95 \pm 0.11$ & $6.92 \pm 0.20$ & $634 \pm 13$ & $1005 \pm 23$ & $21.38 \pm 1.08$ & 4.68 & & $37.1 \pm 1.3$ \\
\hline G3 & NP2 & 3 & $7.50 \pm 0.09$ & $4.43 \pm 0.40$ & $1553 \pm 47$ & $4530 \pm 120$ & $31.35 \pm 2.33$ & 5.25 & & $23.6 \pm 0.9$ \\
\hline G4 & NP6 & & $7.17 \pm 0.08$ & $4.03 \pm 0.25$ & $1338 \pm 32$ & $4387 \pm 114$ & $27.12 \pm 1.58$ & 5.03 & & $33.7 \pm 1.4$ \\
\hline
\end{tabular}

Notes: Data are represented as mean \pm S.D., $\mathrm{n}=3$.

Abbreviations: NFX, norfloxacin; PLGA, poly(lactic-co-glycolic acid); NPs, nanoparticles; HPMC, Hydroxypropyl methylcellulose; K4M; $\mathrm{\eta}$, viscosity. 
diluted hydrogels $(1: 10)$ were measured in triplicates $(\mathrm{pH}$ meter; Griffin and George Ltd., London, England).

\section{Spreadability}

The spreadability of the gels was determined using 2 glass slides. The gel $(0.5 \mathrm{~g})$ was placed over one of them, while the other slide was placed over so that the gel was sandwiched between them. A definite weight was placed over the upper slide and was left for 5 minutes so that gel was pressed uniformly to form a thin layer and no more spreading was expected. The traveled distance by the gel was determined. ${ }^{34}$

\section{Rheology}

The rheological behaviors of the hydrogels were evaluated at different shear rates using a cone and plate digital viscometer (DV-III Programmable Brookfield Rheometer, Stoughton, MA) fitted with a cone spindle \#52. About $0.5 \mathrm{gm}$ of each hydrogel was applied to the plate, and the shear rate was increased gradually in a suitable range to give torque values between 0.1 to $240 \mathrm{rpm}$, with $30 \mathrm{sec}$ between each 2 successive points. The flow pattern of each hydrogel was estimated in triplicates by plotting the shear rate vs shear stress values. The rheological data $\left(\eta_{\min }, \eta_{\max }\right.$, Farrow's constant, and hysteresis loop area) were estimated. Furthermore, Farrow's equation (3) was applied to explore the flow behavior of the gels. ${ }^{26}$

$$
\log \mathrm{D}=\mathrm{N} \log \mathrm{S}-\log \eta
$$

where; $\mathrm{D}$ is the shear rate $\left(\mathrm{sec}^{-1}\right), \mathrm{S}$ is the shear stress (dyne/ $\mathrm{cm}^{2}$ ), $\eta$ is the viscosity (cp) and $N$ (Farrow's constant) is the slope of $\log \mathrm{D}$ against $\log \mathrm{S}$ plot.

\section{In vitro Drug Release Studies}

The in vitro release studies of NFX from the drug-loaded (0.3\%) PLGA NP-laden hydrogels were conducted using semi-permeable membrane tubing, in triplicates, as noted before for drug-loaded NPs. The mean ( \pm S.D.) drug percentages released were plotted vs time. The $\mathrm{Q}_{30 \mathrm{~min}}$ and $\mathrm{Q}_{12 \mathrm{~h}}$ were calculated and compared with those of the optimum NPs formulae (NP2 and NP6).

\section{Ex vivo Transcorneal Drug Permeation Studies}

The ex vivo corneal drug permeation studies of NFX from the drug-loaded $(0.3 \%)$ PLGA NP-laden hydrogels were conducted using the excised goat corneas, in triplicates, as noted before for drug-loaded NPs. The mean ( \pm S.D.) drug percentages permeated were plotted vs time. The $\mathrm{P}_{30 \text { min }}$ and $\mathrm{P}_{12 \mathrm{~h}}$ were calculated and compared with those of the optimum NPs formulae (NP2 and NP6).

\section{Microbiological Studies}

\section{Preparation of Studied Strain}

The bacterial strain was identified and prepared in sterile saline as follows, Pseudomonas aeruginosa strain ATTC 27,853 was obtained to prepare a cell suspension in sterile normal saline. The latter was adjusted to 0.5 McFarland units and the suspension was inoculated to give a final concentration of $10^{5} \mathrm{CFU} / \mathrm{mL}$. This suspension was used for in vitro susceptibility testing.

\section{In vitro Antibacterial Studies}

The antibacterial activity of the freshly prepared formulae was tested with the agar cup (well) diffusion method against Pseudomonas aeruginosa strain. Fixed volumes of the nutrient agar $(20 \mathrm{~mL})$ were seeded with the test microorganism $(0.2 \mathrm{~mL})$ and then, were allowed to solidify in Petri plates. Cups of $10 \mathrm{~mm}$ in diameter were punched aseptically in the plates. One hundred $\mu \mathrm{L}$ of each drug-loaded (0.3\%) PLGA NP-laden hydrogel was filled into the cups. After keeping the plates for 4 hours at $25 \pm 0.5{ }^{\circ} \mathrm{C}$, they were incubated for 24 hours at $37{ }^{\circ} \mathrm{C}$. The zones of inhibition were observed.

\section{In vivo Antimicrobial Studies}

The best-achieved systems (G3, G4) were sterilized by filtration via passage through a sterile membrane filter with a pore size of $0.22 \mu \mathrm{m}$ (Millipore type). The in vivo antimicrobial activity studies were performed on 16 rabbits according to the ARVO Statement for the Use of Animals in Ophthalmic and Vision Research. The rabbits (2-2.5 kg) were derived from Kasr Al-Aini animal house, Faculty of medicine, Cairo University. The animals were acclimatized in an air-conditioned room with alternate night and day cycles (12 hours, each) for one week before the beginning of the studies. They received green food and water ad libitum, and were checked daily for any clinically observable abnormalities, including eye infections and/or lesions. The protocol of the studies was approved ( $\mathrm{Ph} \mathrm{06,}$ Jan 2020) by the Research Ethics Committee of the college of pharmaceutical sciences and drug manufacturing, Misr University for Science and Technology, Egypt.

The rabbits were sedated by intramuscular injections of $1 \mathrm{~mL}$ of $\operatorname{Ketalar}^{\circledR}$ (ketamine hydrochloride, $50 \mathrm{mg} / \mathrm{mL}$ ). The conjunctival sacs were washed with $2-3$ drops of Isopto ${ }^{\circledR}$ Fenicol eye drops (chloramphenicol, $0.5 \%$ ) to remove any 
existing pathogens. The rabbits' corneas were marked with a corneal trephine, $7 \mathrm{~mm}$. The surface epithelium was removed by scraping. The spore suspension of the bacterial strain $(100 \mu \mathrm{L}, 500,000$ spores) was inoculated (intrastromal) by inserting a 27-gauge needle into the center of the cornea to a depth of approximately one half of the corneal thickness.

The rabbits were randomly distributed into 4 groups, each group included 4 rabbits. In all groups, the right eyes were inoculated with Pseudomonas aeruginosa while the left eyes served as controls. The members of Group 1 and Group 2 were treated twice daily with G3 and G4, respectively. For comparison, the members of Group 3 were treated four times daily with NFX suspension $(0.3 \%)$. The treatments were initiated 48 hours post-inoculation and continued for 10 days. No treatment was provided to the members of Group 4 (infected group).

The rabbits' eyes were examined daily for signs of infection. Furthermore, the severity of the inflammatory reaction, as well as the depth and size of corneal ulcer, the stromal edema, and the evidence of vascularization were monitored. The recovery of the surface epithelium for each rabbit was recorded. In a parallel line, the healing of the corneal ulcer and the improvement of the epithelial covering was checked using fluorescein dye. The eyes of each rabbit were photographed to explore the signs of improvement if present. It is worth noting that the ophthalmologist was kept blind to the treatment type and the medication.

\section{Histopathological Studies}

By the end of the treatment period, the animals were sacrificed. The corneas were excised at the limbal margin and each cornea was subjected to histopathological evaluation. The tissue samples were fixed and processed as outlined by Culling. ${ }^{35}$ Samples were fixed in $10 \%$ neutral buffered formalin for 72 hours, processed in serial grades of ethanol, cleared in xylene, infiltrated, and embedded into blocks of paraffin wax. Tissue sections ( $5 \mu$ in thickness) were cut (rotatory microtome), mounted on glass slides, and stained by hematoxylin and eosin as a standard stain for general histological examinations. The corneal regions from different samples were examined and imaged using a full HD microscope camera attached to a Leica microscope. The images were processed with Leica application software (Leica Microsystems GmbH, Wetzlar, Germany).

\section{Results and Discussions Development of NFX-Loaded PLGA NPs} NFX-loaded PLGA NPs were prepared via the doubleemulsion/solvent evaporation technique. This technique is commonly used for preparing NPs because it is simple, reproducible, possesses a limited number of controllable process variables, and can be easily scaled up. ${ }^{23,36}$ NFX is slightly soluble in chloroform $\left(5.5 \mathrm{mg} / \mathrm{mL} ; 25^{\circ} \mathrm{C}\right) .{ }^{37} \mathrm{At}$ a dose of $0.3 \%$, the drug and PLGA were dissolved in chloroform. This was expected to increase the possibility of interaction between the drug and the hydrophobic core of the polymer. It could be inferred that NFX would be less likely to move along with chloroform during the rapid escape of the solvent into the external aqueous phase and hence, a high drug EE\% could be expected. ${ }^{38}$ The incorporation of PVA solution $(1.5 \%, \mathrm{w} / \mathrm{v})$ was necessary to develop small particles possessing uniform particle size distributions. ${ }^{39}$ PVA can lower the interfacial tension at the organic phase-water interface, prevent the aggregation of NPs, and/or preserve their integrity. ${ }^{40}$

\section{Factorial Design Analysis}

For the development of NFX-loaded PLGA NPs, one categorical factor (polymer type, A) and one numerical factor (drug: polymer ratio, B) were investigated. The results were analyzed and the final equations (4-10) for the responses $\left(\mathrm{Y}_{1}-\mathrm{Y}_{7}\right)$ were obtained in terms of coded factors as follows,

$$
\begin{gathered}
\mathrm{Y}_{1}(\mathrm{PS})=317.38-100.45 * \mathrm{~A}+51.32 * \mathrm{~B}[1] \\
-26.13 * \mathrm{~B}[2]+24.27 * \mathrm{~B}[3] \\
\mathrm{Y}_{2}(\mathrm{ZP})=-27.70-1.37 * \mathrm{~A}+3.20 * \mathrm{~B}[1] \\
-4.3 * \mathrm{~B}[2]+1.10 * \mathrm{~B}[3] \\
\mathrm{Y}_{3}(\mathrm{EE} \%)=66.16+6.39 * \mathrm{~A}-17.46 * \mathrm{~B}[1] \\
+19.29 * \mathrm{~B}[2]+7.34 * \mathrm{~B}[3] \\
\mathrm{Y}_{4}\left(\mathrm{Q}_{30 \mathrm{~min}}\right)=12.02+2.56 * \mathrm{~A}-3.7 * \mathrm{~B}[1] \\
+1.79 * \mathrm{~B}[2]-1.3 * \mathrm{~B}[3] \\
\mathrm{Y}_{5}\left(\mathrm{Q}_{12 \mathrm{~h}}\right)=45.1+5.07 * \mathrm{~A}-7.62 * \mathrm{~B}[1] \\
+3.21 * \mathrm{~B}[2]-2.57 * \mathrm{~B}[3] \\
+1.79 * \mathrm{~B}[2]-1.25 * \mathrm{~B}[3] \\
\mathrm{Y}_{6}\left(\mathrm{P}_{30 \mathrm{~min}}\right)=17.4+1.97 * \mathrm{~A}+5.21 * \mathrm{~B}[1] \\
+3.78 * \mathrm{~B}[2]--3.07 * \mathrm{~B}[3] \\
\mathrm{Y}_{7}\left(\mathrm{P}_{12 \mathrm{~h}}\right)=42.75+5.04 * \mathrm{~A}+6.96 * \mathrm{~B}[1] \\
+3
\end{gathered}
$$

where, B [1], B [2], and B [3] are the coefficients of the multi-level numerical factor. 
Table 3 The Factorial Design Analysis Data of the Responses of NFX-Loaded PLGA NPs

\begin{tabular}{|c|c|c|c|c|c|c|c|}
\hline Responses & Sum of Squares & Mean Sum of Squares & F value & $\mathbf{R}^{2}$ & Adjusted $\mathbf{R}^{2}$ & Predicted $\mathbf{R}^{2}$ & Adequate Precision \\
\hline Ps & $94,188.875$ & $23,547.2188$ & II 0.564 & 0.993 & 0.984 & 0.952 & $26.47 I I$ \\
\hline ZP & 74.923 & $|8.73|$ & 34.184 & 0.979 & 0.949 & 0.847 & 17.502 \\
\hline $\mathrm{EE} \%$ & 1955.875 & 488.969 & 23.484 & 0.969 & 0.928 & 0.779 & 13.729 \\
\hline $\mathrm{Q}_{30 \min }$ & 124.677 & 31.169 & 16.447 & 0.956 & 0.898 & 0.689 & 11.952 \\
\hline$Q_{12 h}$ & 732.5 & 183.125 & 4.381 & 0.853 & 0.658 & 0.039 & 5.527 \\
\hline$P_{30 \min }$ & 161.058 & 40.265 & 189.826 & 0.996 & 0.991 & 0.972 & 40.929 \\
\hline$P_{12 h}$ & 586.893 & 146.723 & 4.777 & 0.864 & 0.683 & 0.035 & 5.779 \\
\hline
\end{tabular}

Abbreviations: NFX, norfloxacin; PLGA, Poly(lactic-co-glycolic acid); NP, nanoparticle; PS, particle size; ZP, zeta potential, EE\%, drug entrapment efficiency percentage; $Q_{30 \text { min }}$, drug percentage released after 30 minute; $Q_{12 h}$, drug percentage released after 12 hours; $P_{30 \text { min }}$, drug percentage permeated after 30 minutes; $P_{12 h}$, drug percentage permeated after 12 hours.

The $\mathrm{R}^{2}$ values of all variables were relatively high, indicating that the derived data were statistically valid. The model terms are considered significant when the $p$-value is $\leq 0.05$. Table 3 showed that, except for $\mathrm{Q}_{12 \mathrm{~h}}$ and $\mathrm{P}_{12 \mathrm{~h}}$, the predicted and the adjusted $\mathrm{R}^{2}$ values of all responses were in the acceptable agreement since the difference between them was less than $0.2 .^{41}$ High desirable adequate precision ratios were observed in all responses. The conclusions were depicted by the use of polynomial equations after considering the magnitudes of coefficients and the mathematical sign (negative or positive). A negative sign signifies an antagonistic effect whereas a positive one stands for a synergistic influence. ${ }^{42}$

\section{Effect of Formulation Variables on Particle Size}

The smaller the particle size, the larger the surface area, and the faster the in vitro drug release rate. ${ }^{43}$ The ANOVA results showed that the polymer type and drug: polymer ratio had significant effects on particle size with $P$-values of 0.0003 and 0.0161 , respectively. The average particle size for NPs prepared with ester-terminated or acidterminated PLGA polymers ranged from $356.13 \mathrm{~nm}$ (NP1) to $485.05 \mathrm{~nm}$ (NP4) and from $176.07 \mathrm{~nm}$ (NP5) to $256.12 \mathrm{~nm}$ (NP8), respectively (Table 1). It was clear that the NPs prepared with ester-terminated PLGA polymers were larger than the corresponding NPs prepared with acid-terminated PLGA polymers. The latter NPs are more hydrophilic and possess negatively charged carboxylate groups and hence, more interactions with water would be expected to lower the size of NPs. ${ }^{44}$ In a parallel line, the inter-particle electrostatic repulsions between the carboxylate groups would be expected to minimize the possible collisions of NPs and hence, reduce the particle size. ${ }^{44}$
A direct correlation was established between the drug: polymer ratio and the particle size. This correlation held true, regardless of the polymer type. It could be inferred that the use of a higher polymer concentration would increase the viscosity of the polymer solution in the organic phase and hence, would increase the size of NPs. ${ }^{45}$

PDI is a measure of the width (uniformity) of the particle size distribution. A PDI value close to 0 indicates a homogenous dispersion, while a PDI value close to 1 describes an entirely heterogeneous and polydisperse population. The PDI values of all NPs were small $(<$ 0.3 ), indicating good homogeneity and uniform particle size distributions. ${ }^{46}$

\section{Effect of Formulation Variables on Zeta Potential}

Particles' aggregation is less likely to occur if they are positively or negatively charged with a magnitude of $\geq 30$ $\mathrm{mV}^{47}$ The ANOVA results showed that the polymer type had a significant effect on ZP with a $P$-value of 0.0135 . PLGA is a copolymer of lactic acid and glycolic acid whereas, NFX is a quinoline monocarboxylic acid and hence, all NPs possessed negative zeta potential values. It was clear that NPs prepared with acid-terminated PLGA polymers had larger magnitudes of ZP [ranging from -26.51 (NP5) to -33.62 (NP6)] than the corresponding NPs prepared with ester-terminated PLGA polymers [varying between [-22.52 (NP1) to -30.43 (NP2)], possibly due to the presence of the carboxylic acid groups which got deprotonated at the physiological $\mathrm{pH}^{48}$ Compared to positively charged NPs, the negatively charged systems are expected to be less cytotoxic since the former ones can cause cell lysis by disrupting the membrane. $^{15,49}$ No direct correlation was revealed between the drug: polymer ratio and the ZP, confirming 
the non-significant contribution of this factor on $\mathrm{ZP}$. However, it is worth noting that significantly $(P<0.05)$ higher magnitudes of ZP values were achieved with PLGA NPs prepared at a drug: polymer ratio of $1: 1$, regardless of the polymer type.

\section{Effect of Formulation Variables on Drug Entrapment Efficiency}

The ANOVA results confirmed that polymer type and drug: polymer ratio had significant effects on $\mathrm{EE} \%$ with $P$-values of 0.0288 and 0.0119 , respectively. The average $\mathrm{EE} \%$ for NPs prepared with ester-terminated or acidterminated PLGA polymers ranged from 37.80\% (NP1) to $79.24 \%$ (NP2) and from $59.63 \%$ (NP5) to $91.72 \%$ (NP8), respectively (Table 1). The high drug EE percentages of PLGA NPs could be related to the lipophilic nature of the NFX, which has a low affinity to the aqueous phase and tends to remain, along with PLGA, in the organic domain.

It was clear that the NPs prepared with acid-terminated PLGA polymers had higher EE\% than the corresponding NPs prepared with ester-terminated PLGA polymers. This might be attributed to the strong affinity between the positively charged amine group in NFX and the negatively charged carboxylate groups in the polymer chain.

A direct relationship was observed between drug: polymer ratio and $\mathrm{EE} \%$. This correlation is acceptable until a drug: polymer ratio of $1: 1$. According to Rosca et al ${ }^{50}$ the drug $\mathrm{EE} \%$ is affected by the drainage of the entrapped drug during the expulsion of the solvent. At an optimum drug: polymer ratio, the drug $\mathrm{EE} \%$ is improved, possibly due to the extended entanglement of the drug with the polymer chains as well as the slow shrinkage and/or drainage of the entrapped drug during the expulsion of the solvent. A decrease in the drug EE\% was revealed beyond a 1:1 drug: polymer ratio, regardless of the polymer type. This may be due to the more compact polymer coat, which needs a longer time for the precipitation of the polymer. These findings were in line with those reported by Motwani et $\mathrm{al}^{51}$ who revealed an inverse relationship between the polymer (chitosan-alginate) concentration and gatifloxacin $\mathrm{EE} \%$. At higher polymer concentrations, the polymer would be expected to constitute the bulk of NPs' matrix and hence, a lower volume could be available for drug encapsulation. In a parallel line, Zhang et $\mathrm{al}^{52}$ showed that the highly viscous nature of the gelation medium, at higher polymer concentrations, would hinder the encapsulation of bovine serum albumin in chitosanalginate microspheres.

\section{Effect of Formulation Variables on in vitro Drug Release}

The in vitro release studies of NFX from the investigated NPs and an aqueous drug suspension are illustrated in Figure $1 \mathrm{~A}$. It was clear that $\approx 95.5 \%$ of NFX was released from the aqueous suspension within 6 hours, indicating the non-hindered drug diffusion through the dialysis membrane. On contrary, biphasic drug release profiles were revealed with PLGA NPs. Each drug release profile showed an initial burst release followed by a more prolonged drug release phase. The former phase could be attributed to the drug fractions being weakly bound, adsorbed or located near the surface of NPs, during the intense solvent elimination, due to its hydrophobic nature. ${ }^{53}$ The initial burst drug release is beneficial, in terms of antibacterial activity, to rapidly achieve the minimum effective drug concentration. An extended drug release phase was subsequently attained, probably due to the prolonged partitioning of the remaining drug fractions into the external aqueous phase. This phase was needed to maintain an effective drug concentration for a prolonged period. $^{54}$

The $\mathrm{Q}_{30 \mathrm{~min}}$ and $\mathrm{Q}_{12 \mathrm{~h}}$ of the NPs prepared with the acidterminated PLGA polymers were significantly $(P<0.05)$ higher than the corresponding NPs prepared with esterterminated PLGA polymers. The former NPs are smaller in size and hence, a shorter diffusional path length would be encountered to reach the aqueous phase interface. Furthermore, the greater hydrophobicity of the esterterminated PLGA polymers could lower the wetting, hydration and transition of the surface layers from the glassy solid state to the rubbery gelatinous state upon contact with the aqueous phase, thereby, resulting in lowered drug release rates. These findings were in line with those reported by Ravi et $\mathrm{al}^{55}$ who revealed that the polymer with terminal carboxylic groups, RG $502 \mathrm{H}$, showed low initial burst release compared to RG 502. This could be due to the hydrophilicity of polymer which causes favorable interaction between free carboxylic end groups in the polymer chains and protein.

An inverse correlation was established between either $\mathrm{Q}_{30 \text { min }}$ or $\mathrm{Q}_{12 \mathrm{~h}}$ and the drug: polymer ratio. The higher polymer concentration would enhance the formation of viscous gel layers surrounding the microenvironment of NPs. The gel layers would grow slowly as the aqueous 

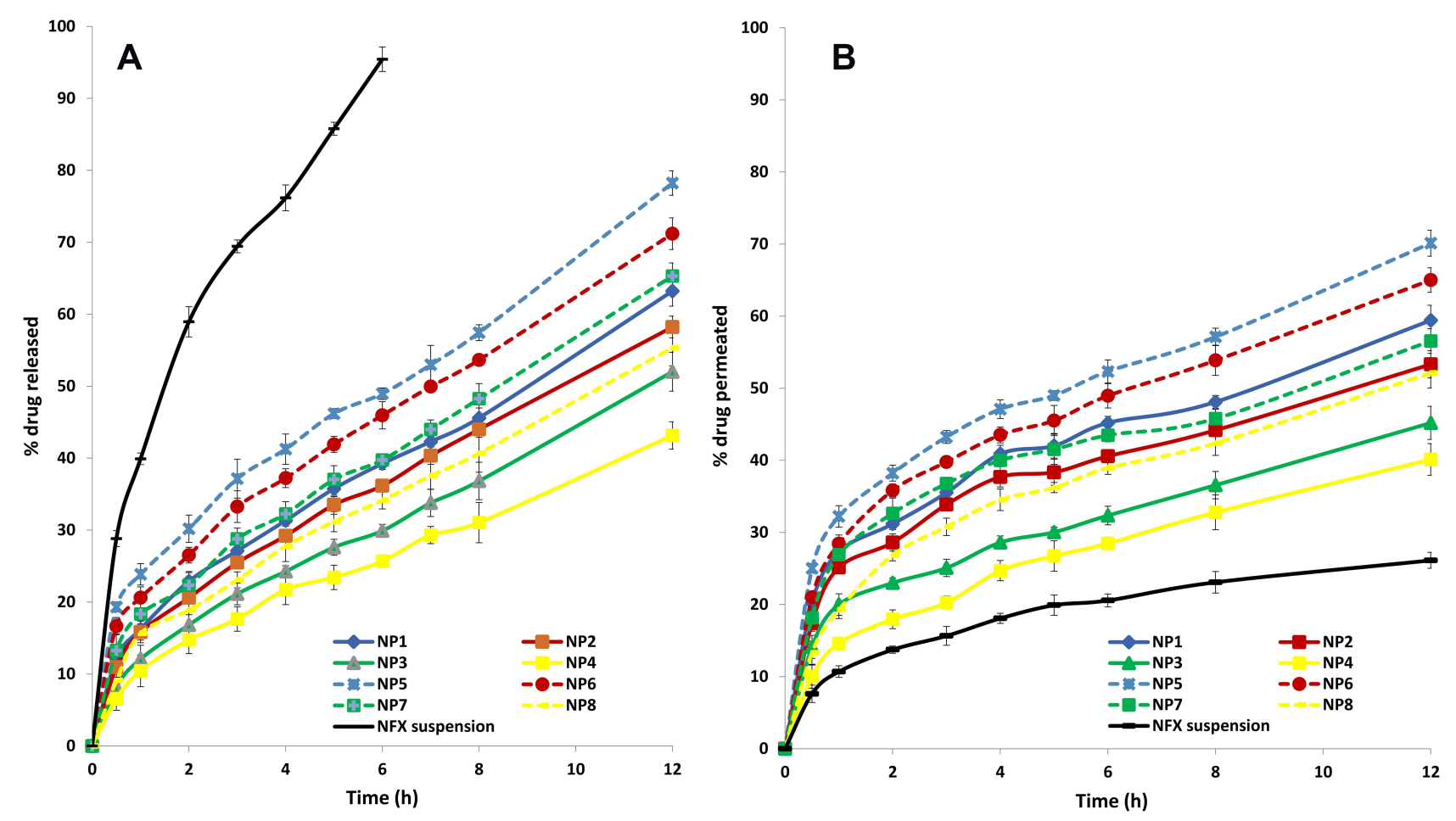

Figure I (A) In vitro release profiles of NFX from NFX-loaded PLGA NPs and NFX suspension in simulated tear fluid ( $\mathrm{pH}$ 7.4), mean \pm S.D., $n=3$. (B) Ex vivo goat corneal permeation profiles of NFX from NFX-loaded PLGA NPs and NFX suspension in simulated tear fluid (pH 7.4), mean \pm S.D., $n=3$.

Abbreviations: NFX, norfloxacin; PLGA, poly(lactic-co-glycolic acid); NPs, nanoparticles.

phase penetrates towards the core. This will gradually increase the thickness of the gel layer and hence, prolong the drug diffusion path length.

\section{Effect of Formulation Variables on ex vivo Corneal Drug Permeation}

The ex vivo corneal drug permeation from NFX suspension and PLGA NPs was studied using goat corneas, Figure 1B. The $\mathrm{P}_{12 \mathrm{~h}}$ of NFX suspension was $26.13 \%$ while the $\mathrm{P}_{12 \mathrm{~h}}$ of NPs ranged from $40.12 \%(\mathrm{NP} 4)$ to $70.12 \%$ (NP5). The favorable permeation of NFX-loaded NPs across the cornea could be attributed to their submicron size and the agglomeration of the NPs in the conjunctival sac and the formation of a depot from which the drug is slowly delivered to the precorneal area. These assumptions were reported for aceclofenac where the goat-corneal uptake of NPs was higher (two-folds) than that of an aqueous drug solution. ${ }^{56} \mathrm{In}$ a parallel line, the rabbit-corneal permeation of flurbiprofen NPs showed enhanced drug permeation (four-folds) over drug solution in phosphate buffer ( $\mathrm{pH}$ 7.4). ${ }^{57}$

The $\mathrm{P}_{30 \mathrm{~min}}$ values of NFX-loaded NPs were higher than the corresponding $\mathrm{Q}_{30 \mathrm{~min}}$ values, showing the drug permeation enhancing effect of NPs. The latter finding could be partly attributed to the high surface area/volume ratio of NPs which would promote a facilitated drug contact with the cornea. Furthermore, the viscosity inducingand permeation enhancing effects of PVA (a non-ionic surfactant) should be also considered. The latter suggestion matches with the findings of Malhotra et $\mathrm{al}^{58}$ who reported that voriconazole ophthalmic drops containing PVA showed higher corneal drug permeation via freshly excised goat corneas, relative to several viscosity inducers.

Interestingly, the $\mathrm{P}_{12 \mathrm{~h}}$ values of NFX-loaded NPs were lower than the corresponding $\mathrm{Q}_{12 \mathrm{~h}}$ values. These latter findings might be related to the simple structure of the dialysis membrane (which acts as a mechanical barrier only) and the complex structure of the cornea (which is made up of lipophilic epithelium, hydrophilic stroma, and lipophilic endothelium). The physicochemical drug properties and the diffusional resistance of the lipophilic and hydrophilic corneal barriers would hinder the corneal drug permeation over time. ${ }^{57}$

The drug permeation profiles from NPs matches with the in vitro drug release profiles. As previously noted for $\mathrm{Q}_{30 \mathrm{~min}}$ and $\mathrm{Q}_{12 \mathrm{~h}}$, the $\mathrm{P}_{30 \mathrm{~min}}$ and $\mathrm{P}_{12 \mathrm{~h}}$ values of NPs prepared with acid-terminated PLGA polymers were significantly $(P<0.05)$ higher than their corresponding NPs prepared with ester-terminated PLGA polymers. In a parallel line, an inverse correlation was observed 
between either $\mathrm{P}_{30 \mathrm{~min}}$ or $\mathrm{P}_{12 \mathrm{~h}}$ and the drug: polymer ratio, regardless of the polymer type.

\section{Corneal Hydration Levels}

The determination of the corneal hydration level is commonly used to evaluate possible corneal damage. The reported hydration level of the normal corneas varies between 76 and $80 \% .{ }^{57}$ Herein, the corneal hydration of all NPs varied between 81.93 (NP6) to $83.12 \%$ (NP4), Table 1. It could be inferred that the developed NPs would not cause any damage to the corneal tissue since a hydration level lower than 3-7\% above the normal value is reported to be harmless. ${ }^{59}$

\section{Selection of the Best Achieved NFX-Loaded PLGA NPs}

The choice of the best-achieved system was based on the transformation of the estimated response (Y1 - Y7) into a desirability index. Based on the set constraints (minimize the PS, $\mathrm{Q}_{30 \mathrm{~min}}$ and $\mathrm{P}_{30 \mathrm{~min}}$, and maximize the magnitude of $\mathrm{ZP}, \mathrm{EE} \%, \mathrm{Q}_{12 \mathrm{~h}}$ and $\mathrm{P}_{12 \mathrm{~h}}$, Table 1), the best achieved esterterminated PLGA NPs (NP2, desirability index 0.6) and the best achieved acid-terminated PLGA NPs (NP6, desirability index 0.57) NP6) were chosen for further studies.

\section{Characterization of the Best Achieved NFX-Loaded PLGA NPs \\ Morphology}

Representative TEM micrographs of NP2 and NP6 were provided in Figure 2A and B, respectively. It was clear that

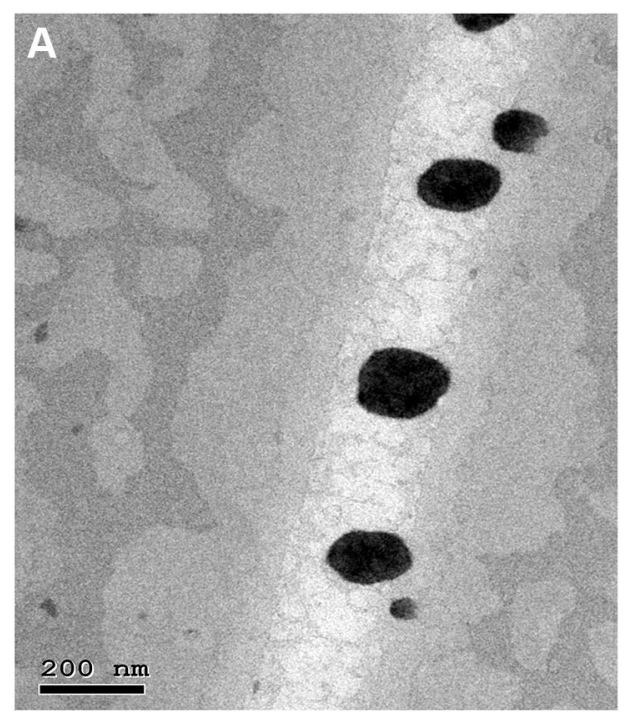

discrete spherical NPs were developed. The non-aggregated tendency of the developed NPs could be related to electrostatic (high magnitude of negative zeta potential) as well as steric (PVA) stabilization. During the evaporation of chloroform, the viscosity of the emulsion would increase and might enhance the coalescence of the emulsion droplets. The incorporation of PVA into the aqueous phase would provide a thin protective film surrounding the droplets and hence, sterically hinder their aggregation and keep their integrity. $^{23,40}$ The NPs appear to have lower particle sizes than those achieved via DLS measurements. This could be related to the possible aggregation of NPs during the estimation of the hydrodynamic diameter. Furthermore, the presence of discrete larger particles in the NPs dispersion could cause greater light scattering and hence, shifts the particle size towards a larger value. The size and shape of NPs could influence their possible ocular irritation potential. The isometric particles possessing obtuse angles and edges, like spherical NPs, are less irritating to the ocular surface than those possessing sharp angles and edges. ${ }^{56}$

\section{Fourier Transform Infrared (FT-IR)}

FT-IR studies were carried out to detect any possible drugpolymer interactions (Figure 3). The FT-IR spectrum of NFX showed a broad characteristic peak between $3500 \mathrm{~cm}^{-1}$ and $3550 \mathrm{~cm}^{-1}$, which could be related to the stretching vibration of -OH group as well as the possible intermolecular hydrogen bonding. The band at $3200 \mathrm{~cm}^{-1}$ to $3300 \mathrm{~cm}^{-1}$ might be attributed to the NH stretching vibration of the imino-moiety of piperazinyl groups. The peak at

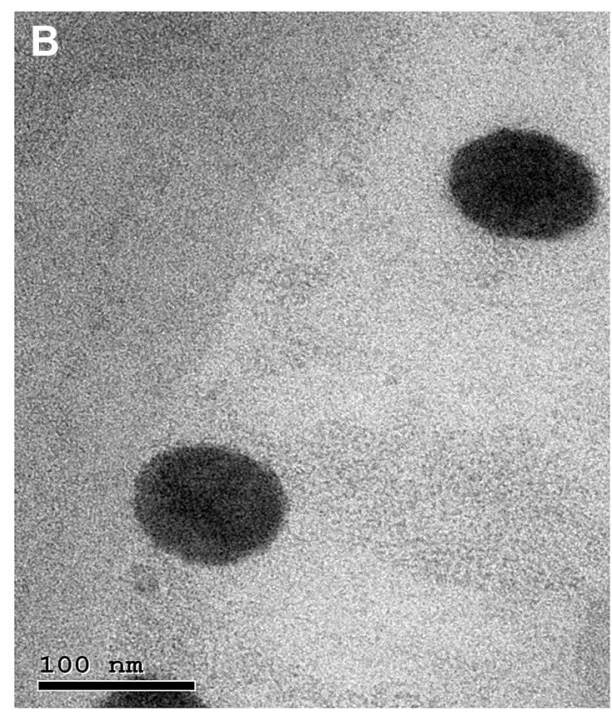

Figure 2 Transmission electron micrographs of NP2 (A) and NP6 (B). Abbreviation: NP, nanoparticles. 

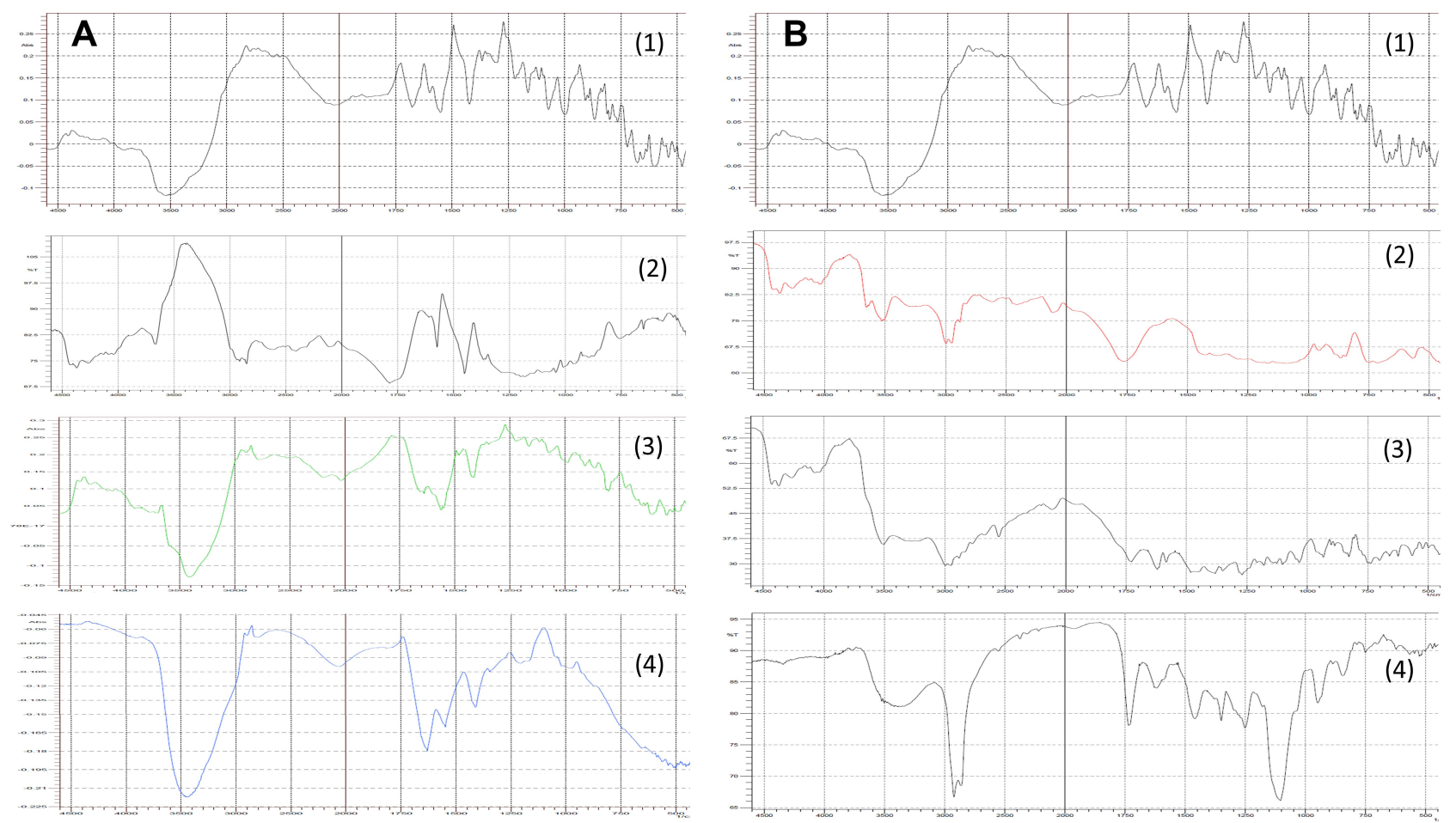

Figure 3 (A) FT-IR spectra of NFX (I), ester terminated PLGA (2), NFX-ester terminated PLGA physical mixture (3) and lyophilized NFX-loaded NP2 (4). (B) FT-IR spectra of NFX (I), acid terminated PLGA (2), NFX-acid terminated PLGA physical mixture (3) and lyophilized NFX-loaded NP6 (4).

Abbreviations: FT-IR, Fourier transform infrared; NFX, norfloxacin; PLGA, poly(lactic-co-glycolic acid); NPs, nanoparticles.

$1670 \mathrm{~cm}^{-1}$ could be ascribed to the $\mathrm{C}=\mathrm{O}$ stretching vibration. The peak at $1550 \mathrm{~cm}-1$ could be referred to as the O-C-O stretching vibration of acid. The peak at $1420 \mathrm{~cm}-1$ was suggested to be attributed to the bending vibration of the O-H group. ${ }^{60}$ The FT-IR spectrum of the ester terminated PLGA showed the - $\mathrm{CH},-\mathrm{CH} 2,-\mathrm{CH} 3$ stretching vibrations between $2850 \mathrm{~cm}^{-1}$ and $2950 \mathrm{~cm}^{-1}$. The band at $1770 \mathrm{~cm}^{-1}$ was assigned to the stretching vibration of $\mathrm{C}=\mathrm{O}$ group that is present in both monomers (lactide and glycolide), while the bands at the range of $1040 \mathrm{~cm}^{-1}-1200 \mathrm{~cm}^{-1}$ were attributed to the C-O stretching vibration (Figure 3A2). These characteristic peaks were comparable to those reported by $\mathrm{Fu}$ et $\mathrm{al}^{61}$ for PLGA. The characteristic peaks of NFX were maintained in the FT-IR spectrum of the drug - polymer physical mixture (Figure 3A3) as well as in the FT-IR spectrum of the lyophilized sample of NP2 (Figure 3A4). Furthermore, no new peaks were developed; suggesting the presence of minimum chemical interactions between NFX and the ester terminated PLGA.

On the other hand, The FT-IR spectrum of the acid terminated PLGA showed broad characteristic peak at $3500 \mathrm{~cm}^{-1}$ which could be related to the stretching vibration of - $\mathrm{OH}$ group of both monomers (Figure 3B2). The $\mathrm{CH},-\mathrm{CH} 2,-\mathrm{CH} 3$ stretching vibrations, the $\mathrm{C}=\mathrm{O}$ stretching vibrations, and the $\mathrm{C}-\mathrm{O}$ stretching vibrations were similarly revealed as noted with the ester terminated PLGA. ${ }^{61}$ The characteristic peaks of NFX were not altered in the FT-IR spectrum of the drug - polymer physical mixture (Figure 3B3). However, the FT-IR spectrum of the lyophilized sample of NP6 (Figure 3B4) showed a broad peak between $3400 \mathrm{~cm}^{-1}$ which could be related to the intermolecular hydrogen bonding between -OH groups of NFX and the acid terminated PLGA. ${ }^{61}$

\section{Development of NFX-Loaded PLGA NP- Laden Hydrogels}

The low ocular absorption of drugs could be attributed to several factors, like the rate of tear turnover and the rapid drainage of the system with a loss of 70 to $80 \%$ of the drug. Moreover, the frequent dosing schedules of eye drops for prolonged periods can negatively influence the eye. ${ }^{62}$ Historically, the ocular ointments were introduced since they possess high viscosities and could not be rapidly drained from the eye. Unfortunately, they usually cause blurred vision and induce poor patient compliance. ${ }^{63}$ In view of the aforementioned, the incorporation of nanostructured particles (NFX-loaded PLGA NPs) in three-dimensional matrices 
(HPMC Hydrogels) was explored as a promising alternative to achieve prolonged ocular drug residence time and achieve good patient compliance.

\section{Characterization of NFX-Loaded PLGA NP-Laden Hydrogels \\ Visual Appearance, Clarity, and $\mathrm{pH}$}

The visual inspection of the systems revealed the development of translucent and homogenous hydrogels with no signs of separation or precipitation. Their $\mathrm{pH}$ values ranged from $6.95(\mathrm{G} 2)$ to $7.50(\mathrm{G} 3)$, Table 2. It could be inferred that these systems are tolerable, would not be expected to cause severe irritation the eye surface.

\section{Spreadability}

The spreadability is an important character of promising hydrogels since it denotes the extent of the area to which the gel readily spreads upon ocular application. The longer the traveled distance, the greater the gel spreadability. ${ }^{34}$ An inverse correlation was established between the polymer concentration and the spreadability, possibly due to the development of stronger polymeric networks. G3 and G4 showed shorter traveled distances than the corresponding gels which were prepared at the lower HPMC concentration (G1 and G2), Table 2.

\section{Rheological Studies}

The viscosity values would influence the flow characteristics of hydrogels upon ocular application. A direct relationship was established between the polymer concentration and the viscosity. The minimum and the maximum viscosity values of G3 and G4 gels were significantly $(P<0.05)$ higher than the corresponding values of G1 and G2 gels, Table 2. The shear stress vs shear rate plots of the systems were depicted in Figure 4. The Farrow's constant $(\mathrm{N})$ values were $>1$ in all systems, ranging from $4.68(\mathrm{G} 2)$ to 5.03 (G4), Table 2. It could be inferred that the shear-thinning pseudoplastic flow was dominating. This rheological behavior would be expected to promote uniform drug distribution over the corneal surface.

A direct correlation was observed between the polymer concentration and the hysteresis loop area. The hysteresis loop areas varied from $21.03 \mathrm{~cm}^{2}$ (G1) to $31.35 \mathrm{~cm}^{2}(\mathrm{G} 3)$, Table 2. As shown in Figure 4, all systems showed good thixotropic behavior. This could be characterized by the progressive rupture of the internal polymeric networks by
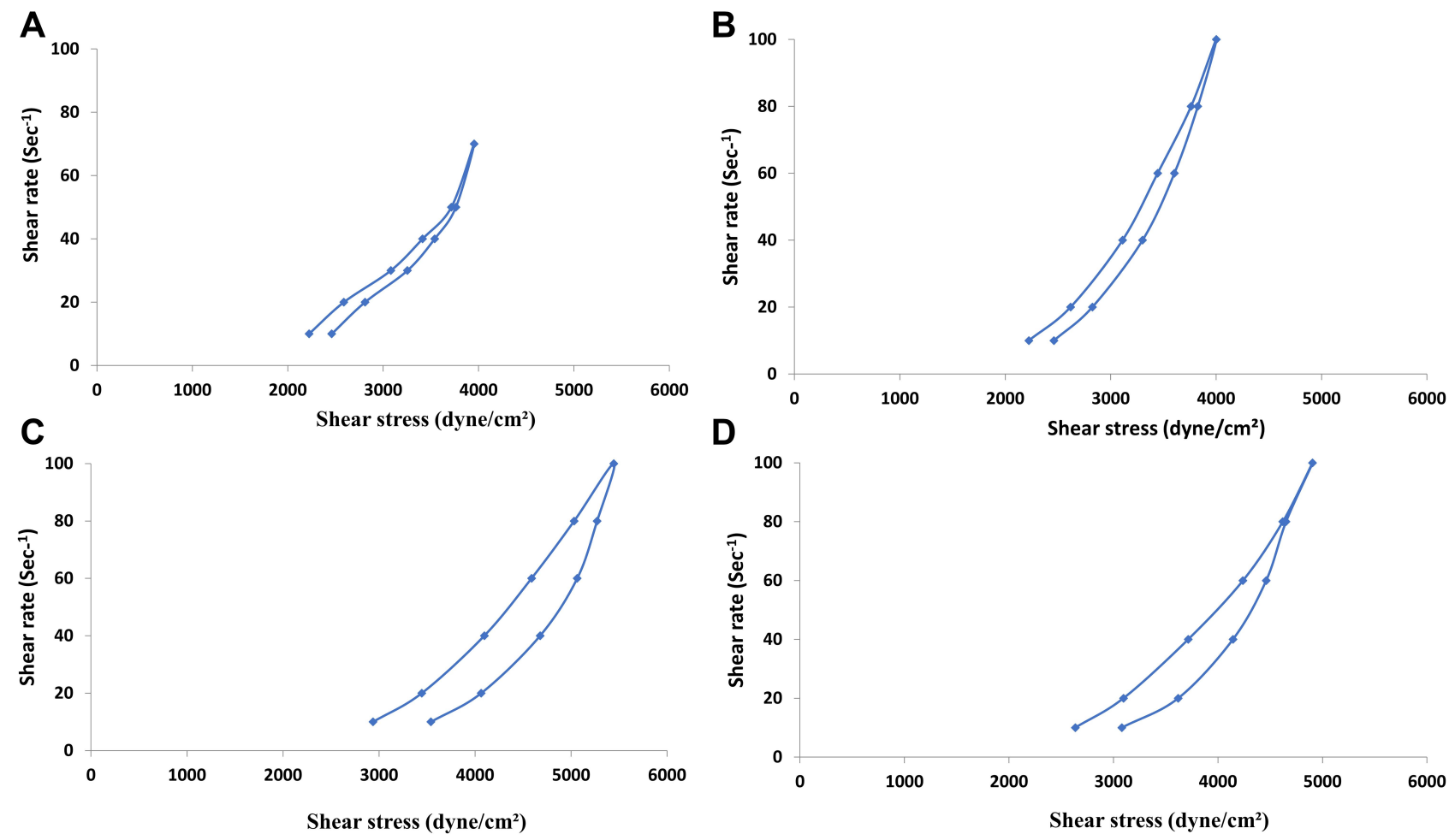

Figure 4 Thixotropic behavior of NFX-loaded PLGA NP-laden HPMC hydrogels (GI (A), G2 (B), G3 (C) and G4 (D). Abbreviations: NFX, norfloxacin; PLGA, poly(lactic-co-glycolic acid); NPs, nanoparticles; HPMC, hydroxypropyl methylcellulose K4M. 
increasing shear and the subsequent reconstruction due to Brownian movement. ${ }^{64}$

\section{In vitro Drug Release Studies}

The in vitro release profiles of NFX from the investigated hydrogels in simulated tear fluid ( $\mathrm{pH}$ 7.4) were represented in Figure 5A. Compared to $\mathrm{Q}_{30 \mathrm{~min}}$ and $\mathrm{Q}_{12 \mathrm{~h}}$ of NP2 and NP6, the corresponding values of the developed hydrogels were significantly $(P<0.05)$ lower, confirming the development of more prolonged drug release profiles. When HPMC-based hydrogels got exposed to an aqueous medium (STF), the polymer hydrates to form a gel layer and the surface layers transition from a glassy solid state to a rubbery gelatinous state. The gel core, containing the NPs, remains essentially dry at this stage. The drug on the surface of NPs is likely to pass into the solution rapidly providing an initial pulse of drug. As the fluid penetrates towards the NPs, the gel layer grows progressively and the diffusion path length for the drug is prolonged. When the full hydration of the outer layer is achieved, the polymer chain relaxation can produce a loss of integrity, disentanglement, and matrix erosion, allowing for the subsequent prolonged drug release from NPs. ${ }^{65}$

An inverse correlation could be established between HPMC concentration and $\mathrm{Q}_{30 \mathrm{~min}}$ or $\mathrm{Q}_{12 \mathrm{~h}}$. It could be inferred that the higher density of polymer chains in the gels' microstructures would allow a more retarded diffusion path length for the drug. ${ }^{66}$

\section{Ex vivo Transcorneal Drug Permeation Studies}

The ex vivo transcorneal drug permeation profiles of NFX from the investigated hydrogels in simulated tear fluid $(\mathrm{pH}$ 7.4) were represented in Figure 5B. Compared to $P_{30 \mathrm{~min}}$ and $\mathrm{P}_{12 \mathrm{~h}}$ of NP2 and NP6, it was clear that the corresponding percentages of the developed hydrogels were significantly $(P<0.05)$ lower. These findings could be related to the mucoadhesive and viscosity-inducing properties of HPMC which can markedly prolong the drug residence time in the conjunctival sac and allow more time for the drug to diffuse via the ocular layers. The advantages of the incorporation of nanostructured systems in HPMC ocular systems were previously reported for many drugs like levofloxacin hemihydrate, ${ }^{67}$ acetazolamide, ${ }^{68}$ and voriconazole. ${ }^{69}$

\section{Microbiological Studies}

\section{In vitro Antimicrobial Studies}

All formulae were tested microbiologically by the agar cup (well) diffusion method. Clear zones of inhibition were

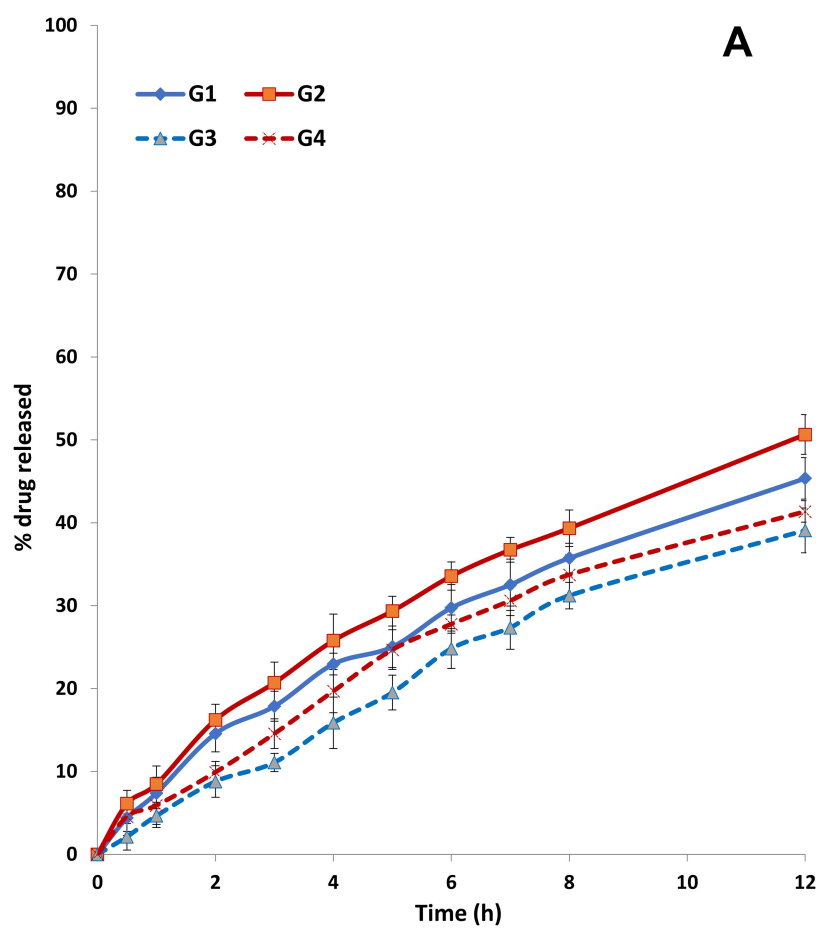

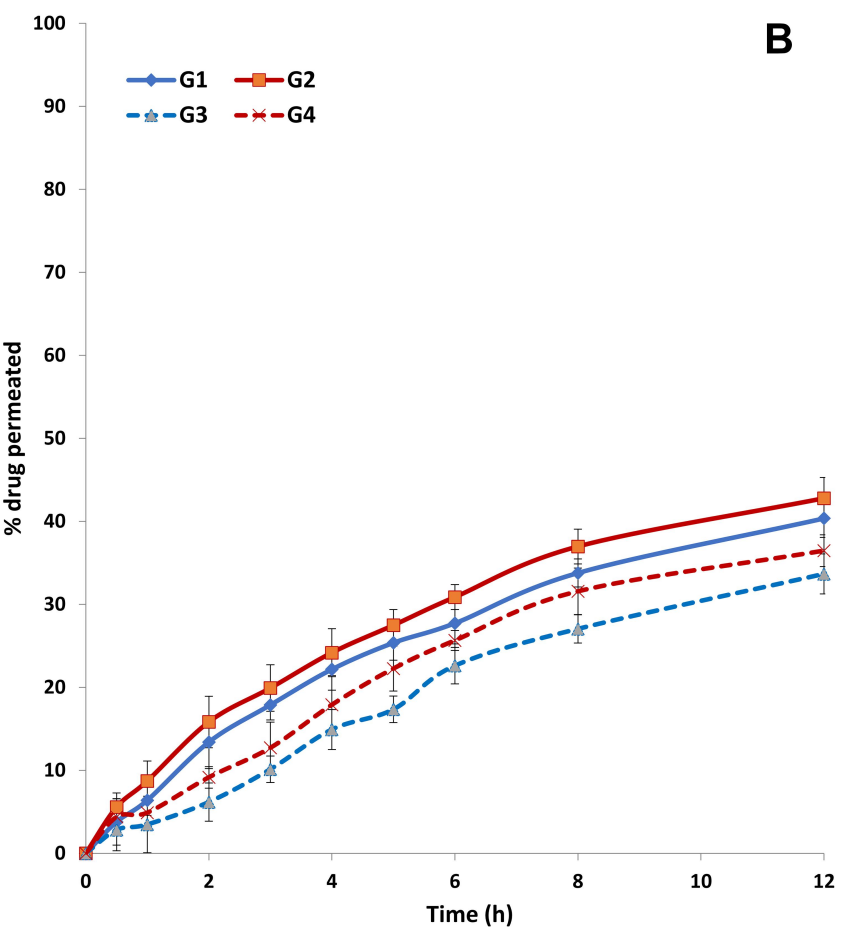

Figure 5 (A) In vitro release profiles of NFX from NFX-loaded PLGA NP-laden HPMC hydrogels in simulated tear fluid $(\mathrm{pH} 7.4)$, mean \pm S.D., $n=3$. (B) Ex vivo goat corneal permeation profiles of NFX from NFX-loaded PLGA NP-laden HPMC hydrogels in simulated tear fluid $(\mathrm{pH} 7.4)$, mean \pm S.D., $n=3$.

Abbreviations: NFX, norfloxacin; PLGA, poly(lactic-co-glycolic acid), NPs, nanoparticles; HPMC, Hydroxypropyl methylcellulose K4M. 
obtained, ranging in diameter from $23.6 \mathrm{~mm} \mathrm{(G3)} \mathrm{to}$ $37.1 \mathrm{~mm}(\mathrm{G} 2)$ after 24 hours, Table 2. It was clear that the acid-terminated PLGA NP6-laden hydrogels (G2 and G4) showed larger zones of inhibition than the corresponding ester-terminated PLGA NP2-laden hydrogels (G1 and G3, respectively). As noted earlier, the former systems showed significantly $(P<0.05)$ higher $\mathrm{Q}_{12 \mathrm{~h}}$ values. By extrapolation, these systems would be expected to allow the release of higher drug percentages following an incubation period of 24 hours and hence, this would augment their in vitro antimicrobial activity. The efficacy of these systems against microorganisms could be described with several mechanisms, including, (i) the mucoadhesive and viscosity-inducing properties of HPMC hydrogels, (ii) the fusion of NPs with the microbial cell wall or membrane and the release of the loaded drug within the microorganism, and (iii) the adsorption of NPs to the cell wall, where they serve as a reservoir for the continuous diffusion of the drug into the microorganism, and (iv) the greater stability of the encapsulated drug in NPs against rapid enzymatic/ hydrolytic degradation. ${ }^{70}$

Taking into consideration the main goal of achieving more prolonged transcorneal drug permeation rates via thixotropic systems possessing promising antimicrobial activities, G3 and G4 systems were promoted for further in vivo studies.

\section{In vivo Antimicrobial Studies}

All rabbits' eyes demonstrated severe clinical inflammatory features in the form of conjunctival hyperemia and corneal opacity due to stromal edema with overlying epithelial defect (corneal ulcer) post-infection with Pseudomonas aeruginosa. The mean rank of opacity scores of all infected rabbits showed non-significant $(P>$ 0.05 ) differences at this time, as all groups showed dense opacity covering the entire anterior segment. The rabbits started to receive treatments after 48 hours postinoculation. The rabbits' eyes were examined daily using a hand-held portable slit-lamp biomicroscope for the assessment of the severity of the inflammatory reaction, size, and depth of corneal ulcer, stromal edema, and evidence of vascularization.

Figure 6 shows the conjunctival hyperemia and corneal edema in rabbits with Pseudomonas-induced keratitis pre(A) and 10 days post- (B) non-treatment in the infected group (a) or treatment in drug suspension- (b), G3- (c), G4- (d) groups. All rabbits' eyes demonstrated severe conjunctival hyperemia and corneal opacity (stromal edema) post-infection with Pseudomonas aeruginosa
A
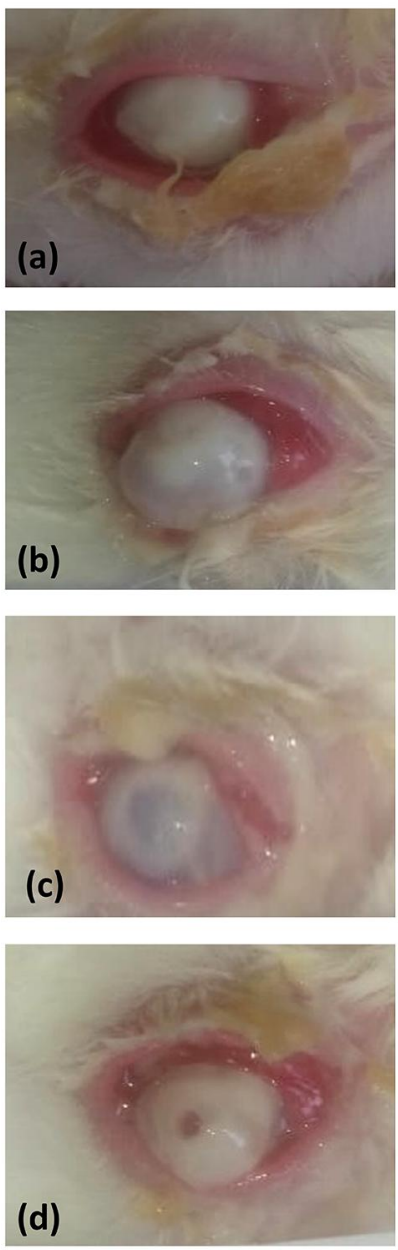

Figure 6 Conjunctival hyperemia and corneal edema in rabbits with Pseudomonasinduced keratitis pre- (A) and 10 days post- (B) non-treatment in the infected group (a) or treatment in drug suspension- (b), G3- (c), G4- (d) groups.

before starting treatment, Figure 6A. The corneal opacity was scored and graded as follows, grade 0 , eyes are identical to their original state before the infection; grade 1 , faint opacity in the infected area; grade 2 , dense opacity in the infected area; grade 3 , dense opacity covering the entire anterior segment; grade 4, perforation of the cornea, ${ }^{71}$ Table 4.

By the end of the treatment period, the corneal opacities in drug suspension-, G3-, G4-groups were better and showed significantly lower grades than those observed in the infected-group, Figure 6B. As noted in Table 4, grade 1 corneal opacity was revealed in drug suspension-, G3- and G4-groups, as compared to grade 3 in the infected-group. This was statistically evaluated via Mann Whitney $U$-test at $P<0.05$. In a parallel line, the sizes of ulcers in drug suspension-, G3- and G4-groups were significantly lower 
Table 4 Comparison of the Clinical and Histopathological Findings of the Excised Corneal Tissue Sections in the Investigated Groups

\begin{tabular}{|l|l|l|l|l|l|}
\hline Points of Comparison & Control Values & \multicolumn{2}{|l|}{ Groups } & \multicolumn{3}{|l|}{} \\
\cline { 3 - 6 } & & Infected & Drug Suspension & G3 & G4 \\
\hline Corneal opacity & - & Grade 3 & Grade I & Grade I & Grade I \\
Ulcer size & - & $9 \mathrm{~mm}$ & $2 \mathrm{~mm}$ & $2 \mathrm{~mm}$ & $3 \mathrm{~mm}$ \\
Conjunctival hyperemia & - & Severe & Severe & Severe & Severe \\
Stromal edema & - & Severe & Moderate & Moderate \\
Covering epithelial damage & - & +++ & + & + & + Moderate \\
Collagen fibers disorganization & - & +++ & ++ & ++ \\
Neovascularization & - & ++ & ++ & ++ \\
Inflammatory cells infiltrate & - & +++ & +++ & ++ \\
\hline
\end{tabular}

Note: Symbols: $(-)$ nil, $(+)$ mild, $(++)$ moderate and $(+++)$ severe.

than those observed in the infected-group. They showed better resolution of the stromal edema, marked decreases in the areas of infiltration, and smaller sizes of ulcers.

\section{Histopathological Studies}

The histopathological examinations of the excised corneal specimens were carried out to evaluate and compare the degree of corneal injuries for the selected NPs-laden hydrogels (G3 and G4)- with those for the control-, the infected-, and drug suspension-groups, Figure 7. The degree of the covering epithelial damage, the collagen fibers disorganization, the neovascularization, and the inflammatory cells infiltrate in all groups were summarized in Table 4. The corneal bacterial infection is suggested to occur in two-steps. The first one involves the interaction of the bacteria with the surface of the epithelial cells of the cornea. The second step involves the bacterial penetration into the stroma where the released toxins would mediate severe tissue inflammation and possible damage. Following intrastromal injections, keratitis is characterized by marked bacterial replication and severe ophthalmic changes like stromal swelling, corneal epithelial cell destruction, and migration of polymorphonuclear leukocytes to the tear film. ${ }^{72}$

The control eyes showed normal histological features of corneal layers with intact covering epithelium (black arrow), underlying well organized stromal collagen lamellar morphology (star) and intact posterior limiting membrane with lining endothelium, Figure 7A. On the other hand, the histopathological examination of the infected eyes (Figure 7B) demonstrated wide areas of detached, denuded and lost covering epithelial layer (black arrow) accompanied with moderate edema of stromal layer and disorganized collagen fibers with many proliferating fibroblasts (green arrow). In conjunction with neovascularization (star), moderate to severe polymorphonuclear inflammatory cells infiltrate were recorded (red arrow). A high grade (3) of corneal opacity and a large corneal ulcer $(9 \mathrm{~mm})$ were observed.

Compared to the infected eyes, the histopathological examination of the eyes treated with NFX suspension (Figure 7C), G3 (Figure 7D) or G4 (Figure 7E) showed almost intact epithelium with significant thickening and demonstrated fewer inflammatory cells infiltrate (red arrow), moderate edema, minor changes of collagenous fibers in the stroma, lower degree of congested/dilated blood vessels (star) and less proliferating fibroblasts (green arrow). In comparison to NFX suspension and G4-treated groups, G3-treated group showed a lower degree of congested (dilated) blood vessels, and stromal polymorphonuclear leukocytes infiltrate.

Unlike the traditional ocular preparations of NFX, G3 and G4 systems would allow prolonged pre-corneal drug residence time, better corneal drug permeation, and lower probabilities of inducing corneal drug precipitates. With G3 and G4 systems, prolonged therapeutic drug levels could be maintained with reasonable frequencies of application (twice daily) and fewer side effects, taking into consideration the higher antimicrobial activity of G4 system and the fewer side effects of G3 system.

The limitations of this study include the small sample size and the encountered redness, which may denote irritation, in all treated groups. However, one should consider that the eye burning, itching, irritation and redness are common side effects following NFX instillation.

\section{Conclusions}

NFX-loaded PLGA NPs were successfully developed using the double-emulsion/solvent evaporation technique. Based on the factorial design analysis and estimation of the desirability 

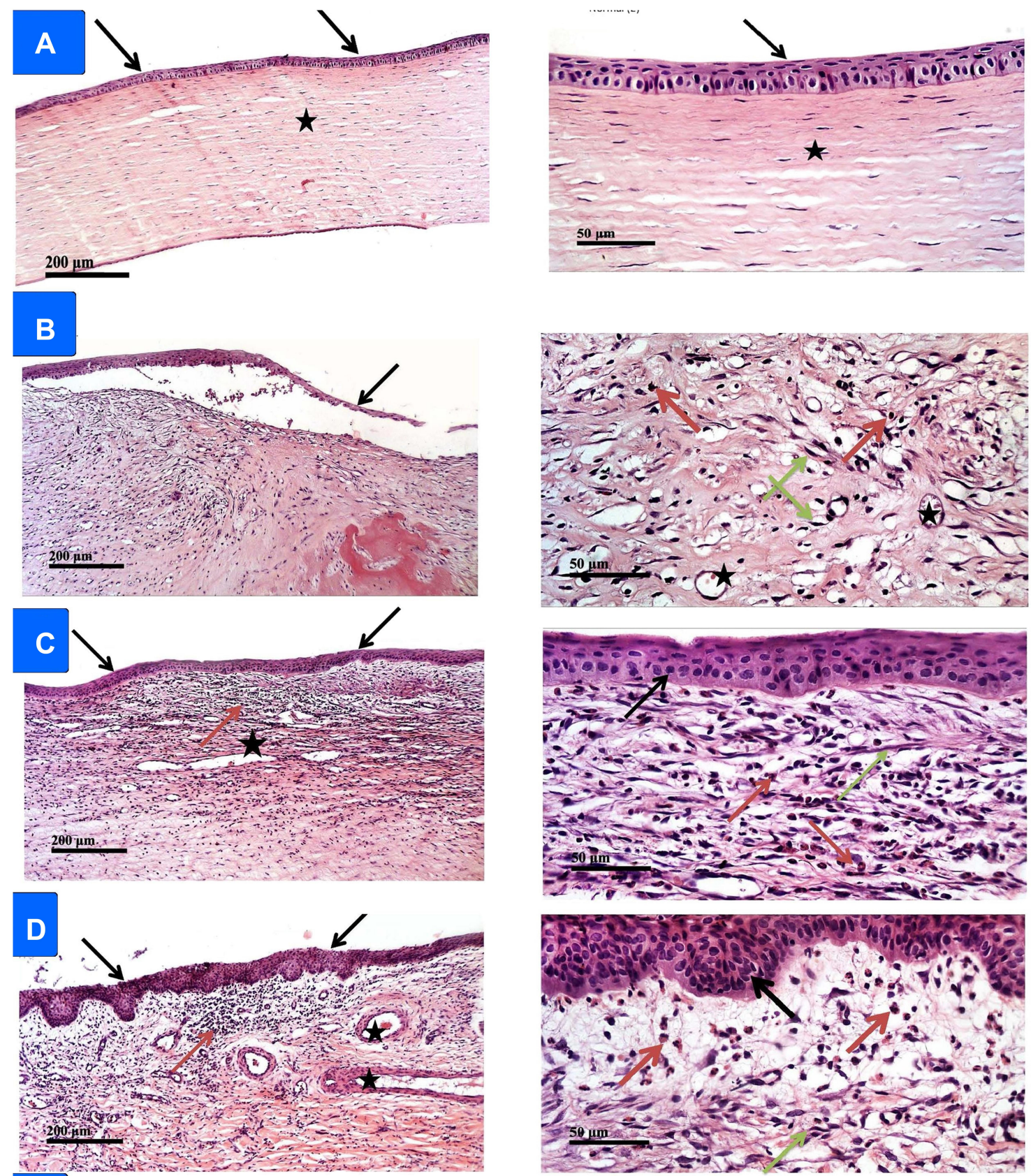

E
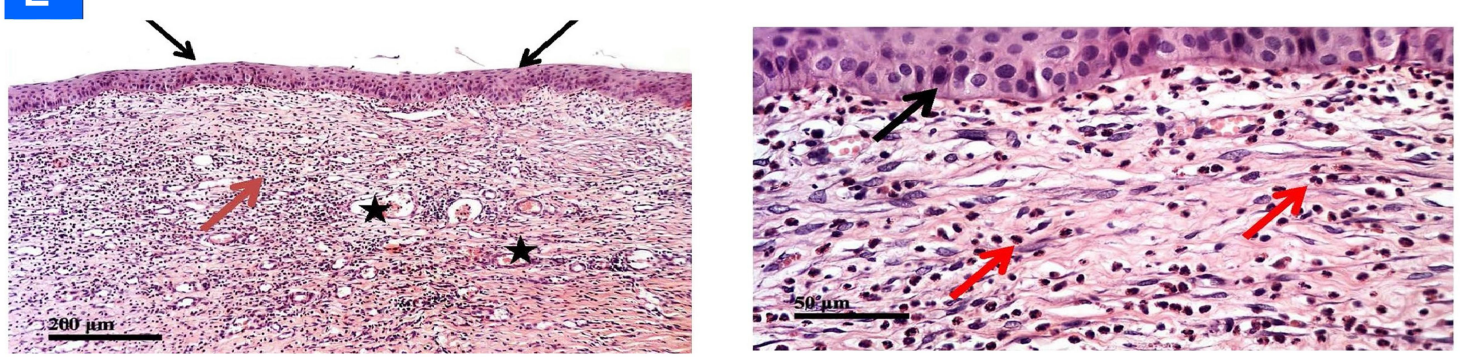

Figure 7 Histopathological changes observed, under two magnifications, in the sections of the excised corneal tissues in different groups [control (A), infected (B), drug suspension (C), G3 (D), G4 (E)]. The black arrow points to the epithelial layer, the green arrow shows the proliferating fibroblasts, and the red arrow refers to the polymorphonuclear inflammatory cells infiltrate. The stars denote the stromal collagen lamellar morphology (A), neovascularization (B), congested/dilated blood vessels (C-E). 
indices, two formulae (NP2 and NP6) were selected since they possess high $\mathrm{ZP}$ magnitude, $\mathrm{EE} \%, \mathrm{Q}_{12 \mathrm{~h}}$ and $\mathrm{P}_{12 \mathrm{~h}}$, as well as, low PS, $\mathrm{Q}_{30 \text { min }}$ and $\mathrm{P}_{30 \mathrm{~min}}$. Clear, spreadable, tolerable, and thixotropic NFX-loaded PLGA NP-laden HPMC-based hydrogels were successfully developed. G3 and G4 systems could be considered as suitable ocular delivery systems for NFX, with respect to prolonged drug permeation profiles, reasonable antimicrobial activities against Pseudomonas aeruginosa and promising safety profiles. Further clinical studies are needed to confirm the potential of these systems.

\section{Disclosure}

The authors report no conflicts of interest for this work.

\section{References}

1. Jumelle C, Gholizadeh S, Annabi N, Dana R. Advances and limitations of drug delivery systems formulated as eye drops. J Control Rel. 2020;321:1-22. doi:10.1016/j.jconrel.2020.01.057

2. Zhang W, Prausnitz MR, Edwards A. Model of transient drug diffusion across cornea. J Control Rel. 2004;99:241-258. doi:10.1016/j. jconrel.2004.07.001

3. Agarwal P, Scherer D, Günther B, Rupenthal ID. Semifluorinated alkane based systems for enhanced corneal penetration of poorly soluble drugs. Int J Pharm. 2018;538(1-2):119-129. doi:10.1016/j. ijpharm.2018.01.019

4. Lakhundi S, Siddiqui R, Khan NA. Pathogenesis of microbial keratitis. Microb Pathog. 2017;104:97-109.

5. Araújo J, Vega E, Lopes C, Egea MA, Garcia ML, Souto EB. Effect of polymer viscosity on physicochemical properties and ocular tolerance of FB-loaded PLGA nanospheres. Colloids Surf B Biointerfaces. 2009;72(1):48-56. doi:10.1016/j.colsurfb.2009.03.028

6. Cañadas $\mathrm{C}$, Alvarado $\mathrm{H}$, Calpena $\mathrm{AC}$, et al. In vitro, ex vivo and in vivo characterization of PLGA nanoparticles loading pranoprofen for ocular administration. Int $J$ Pharm. 2016;511(2):719-727. doi:10.1016/j.ijpharm.2016.07.055

7. Onlen Y, Tamer C, Oksuz H, et al. Comparative trial of different anti-bacterial combinations with propolis and ciprofloxacin on Pseudomonas keratitis in rabbits. Microbiol Res. 2007;162 (1):62-68. doi:10.1016/j.micres.2006.07.004

8. Constantinou M, Daniell M, Snibson GR, Vu HT, Taylor HR. Clinical efficacy of moxifloxacin in the treatment of bacterial keratitis: a randomized clinical trial. Ophthalmology. 2007;114(9):1622-1629. doi:10.1016/j.ophtha.2006.12.011

9. Ubani-Ukoma U, Gibson D, Schultz G, Silva BO, Chauhan A. Evaluating the potential of drug eluting contact lenses for treatment of bacterial keratitis using an ex vivo corneal model. Int $J$ Pharm. 2019;565:499-508. doi:10.1016/j.ijpharm.2019.05.031

10. Goldstein EJ. Norfloxacin, a fluoroquinolone antibacterial agent. Classification, mechanism of action, and in vitro activity. Am J Med. 1987;82(6):3-17. doi:10.1016/0002-9343(87)90612-7

11. Lin HH, Ko SM, Hsu LR, Tsai YH. The preparation of norfloxacin-loaded liposomes and their in-vitro evaluation in pig's eye. J Pharm Pharmacol. 1996;48(8):801-805. doi:10.1111/j.2042-7158.1996.tb03977.x

12. Kalyanwat R, Shrivastava B, Pathak K. Bioadhesive ocular inserts of Norfloxacin for the treatment of ocular E. coli infection: development and in vitro evaluation. Pharm Chem J. 2017;4(4):47-56.

13. Liu S, Dozois MD, Chang CN, et al. Prolonged ocular retention of mucoadhesive nanoparticle eye drop formulation enables treatment of eye diseases using significantly reduced dosage. Mol Pharm. 2016;13 (9):2897-2905. doi:10.1021/acs.molpharmaceut.6b00445
14. Öztürk AA, Yenilmez E, Özarda MG. Clarithromycin-loaded poly (lactic-co-glycolic acid)(PLGA) nanoparticles for oral administration: effect of polymer molecular weight and surface modification with chitosan on formulation, nanoparticle characterization and antibacterial effects. Polymers. 2019;11(10):1632. doi:10.3390/polym11101632

15. Mundargi RC, Babu VR, Rangaswamy V, Patel P, Aminabhavi TM. Nano/micro technologies for delivering macromolecular therapeutics using poly (D, L-lactide-co-glycolide) and its derivatives. J Control Rel. 2008;125(3):193-209. doi:10.1016/j.jconrel.2007.09.013

16. Silva AT, Cardoso BC, Silva ME, et al. Synthesis, characterization, and study of PLGA copolymer in vitro degradation. $J$ Biomater Nanobiotechnol. 2015;6(1):8-19. doi:10.4236/jbnb.2015.61002

17. Alkholief M, Albasit H, Alhowyan A, et al. Employing a PLGA-TPGS based nanoparticle to improve the ocular delivery of Acyclovir. Saudi Pharm J. 2019;27(2):293-302. doi:10.1016/j.jsps. 2018.11.011

18. Allison SD. Effect of structural relaxation on the preparation and drug release behavior of poly(lactic-co-glycolic) acid microparticle drug delivery systems. J Pharm Sci. 2008;97(6):2022-2035. doi:10. 1002/jps.21124

19. Zhang L, Pornpatt Ananangkul D, Hu CHJ, et al. Development of nanoparticles for antimicrobial drug delivery. Curr Med Chem. 2010;17:585-594. doi:10.2174/092986710790416290

20. Ahmed TA, Aljaeid BM. A potential in situ gel formulation loaded with novel fabricated poly(lactide-co-glycolide) nanoparticles for enhancing and sustaining the ophthalmic delivery of ketoconazole. Int J Nanomedicine. 2017;12:1863-1875. doi:10.2147/IJN.S131850

21. Gonzalez-Pizarro R, Carvajal-Vidal P, Halbault Bellowa L, Calpena AC, Espina M, García ML. In-situ forming gels containing fluorometholone-loaded polymeric nanoparticles for ocular inflammatory conditions. Colloids Surf B Biointerfaces. 2019;175: 365-374. doi:10.1016/j.colsurfb.2018.11.065

22. Abrego G, Alvarado H, Souto EB, et al. Biopharmaceutical profile of pranoprofen-loaded PLGA nanoparticles containing hydrogels for ocular administration. Eur J Pharm Biopharm. 2015;95(Pt B):261-270. doi:10.1016/j.ejpb.2015.01.026

23. Park MH, Jun HS, Jeon JW, et al. Preparation and characterization of bee venom loaded PLGA particles for sustained release. Pharm Dev Technol. 2018;23(9):857-864. doi:10.1080/10837450.2016.1264415

24. Abd-Elbary A, Makky AM, Tadros MI, Alaa-Eldin AA. Laminated sponges as challenging solid hydrophilic matrices for the buccal delivery of carvedilol microemulsion systems: development and proof of concept via mucoadhesion and pharmacokinetic assessments in healthy human volunteers. Eur J Pharm Sci. 2016;82:31-44. doi:10.1016/j.ejps.2015.11.006

25. Yeap SP, Lim J, Ngang HP, Ooi BS, Ahmad AL. Role of particle-particle interaction towards effective interpretation of z-average and particle size distributions from Dynamic Light Scattering (DLS) analysis. J Nanosci Nanotechnol. 2018;18 (10):6957-6964. doi:10.1166/jnn.2018.15458

26. Tayel SA, El-Nabarawi MA, Tadros MI, Abd-Elsalam WH. Promising ion-sensitive in situ ocular nanoemulsion gels of terbinafine hydrochloride: design, in vitro characterization and in vivo estimation of the ocular irritation and drug pharmacokinetics in the aqueous humor of rabbits. Int J Pharm. 2013;443(1-2):293-305. doi:10.1016/j.ijpharm.2012.12.049

27. Yehia SA, El-Ridi MS, Tadros MI, El-Sherif NG. Phenylalanine-free taste-masked orodispersible tablets of fexofenadine hydrochloride: development, in vitro evaluation and in vivo estimation of the drug pharmacokinetics in healthy human volunteers. Pharm Dev Technol. 2015;20(5):528-539. doi:10.3109/10837450.2014.882942

28. Sah AK, Suresh PK, Verma VK. PLGA nanoparticles for ocular delivery of loteprednol etabonate: a corneal penetration study. Artif Cells Nanomed Biotechnol. 2017;45(6):1156-1164. doi:10.1080/ 21691401.2016.1203794 
29. Yadav M, Ahuja M. Preparation and evaluation of nanoparticles of gum cordia, an anionic polysaccharide for ophthalmic delivery. Carbohydr Polym. 2010;81:871-877. doi:10.1016/j.carbpol.2010. 03.065

30. Monti D, Chetoni P, Burgalassi S, Najarro M, Saettone MF. Increased corneal hydration induced by potential ocular penetration enhancers: assessment by differential scanning calorimetry (DSC) and by desiccation. Int J Pharm. 2002;232(1-2):139-147. doi:10.1016/S037 8-5173(01)00907-3

31. Mazyed EA, Abdelaziz AE. Fabrication of transgelosomes for enhancing the ocular delivery of acetazolamide: statistical optimization, in vitro characterization, and in vivo study. Pharmaceutics. 2020;12(5):465. doi:10.3390/pharmaceutics1205 0465

32. Abdellatif MM, Khalil IA, Khalil MAF. Sertaconazole nitrate loaded nanovesicular systems for targeting skin fungal infection: in-vitro, ex-vivo and in-vivo evaluation. Int J Pharm. 2017;527(1-2):1-11. doi:10.1016/j.ijpharm.2017.05.029

33. Almutairi MS, Ali M. Direct detection of saponins in crude extracts of soapnuts by FTIR. Nat Prod Res. 2015;29(13):1271-1275. doi:10.1080/14786419.2014.992345

34. Soliman SM, Abdel Malak NS, El-Gazayerly ON, Abdel Rehim AA. Formulation of microemulsion gel systems for transdermal delivery of celecoxib: in vitro permeation, anti-inflammatory activity and skin irritation tests. Drug Discov Ther. 2010;4(6):459-471.

35. Culling CF. Handbook of Histopathological and Histochemical Techniques: Including Museum Techniques. 3rd ed. Butterworth; 1974.

36. Kim BK, Hwang SJ, Park JB, Park HJ. Preparation and characterization of drug-loaded polymethacrylate microspheres by an emulsion solvent evaporation method. J Microencapsul. 2002;19(6):811-822. doi:10.1080/0265204021000022770

37. O'Neil MJ, ed. The Merck Index: An Encyclopedia of Chemicals, Drugs, and Biologicals. Whitehouse Station, NJ: Merck and Co., Inc; 2006.

38. Meng FT, Ma GH, Liu YD, Qiu W, Su ZG. Microencapsulation of bovine hemoglobin with high bio-activity and high entrapment efficiency using a W/O/W double emulsion technique. Colloids Surf B Biointerfaces. 2004;33(3-4):177-183. doi:10.1016/j.colsurfb.2003. 10.003

39. Wang H, Jia Y, Hu W, Jiang H, Zhang J, Zhang L. Effect of preparation conditions on the size and encapsulation properties of mPEG-PLGA nanoparticles simultaneously loaded with vincristine sulfate and curcumin. Pharm Dev Technol. 2013;18(3):694-700. doi:10.3109/10837450.2012.696267

40. Mobarak DH, Salah S, Elkheshen SA. Formulation of ciprofloxacin hydrochloride loaded biodegradable nanoparticles: optimization of technique and process variables. Pharm Dev Technol. 2014;19 (7):891-900. doi:10.3109/10837450.2013.836293

41. Alexander DLJ, Tropsha A, Winkler DA. Beware of $\mathrm{R}^{(2)}$ : simple, unambiguous assessment of the prediction accuracy of QSAR and QSPR models. J Chem Inf Model. 2015;55(7):1316-1322. doi:10.10 21/acs.jcim.5b00206

42. Sudhakar B, Krishna MC, Murthy KV. Factorial design studies of antiretroviral drug-loaded stealth liposomal injectable: pEGylation, lyophilization and pharmacokinetic studies. Appl Nanosci. 2016;6 (1):43-60. doi:10.1007/s13204-015-0408-8

43. Tadros MI, Al-Mahallawi AM. Long-circulating lipoprotein-mimic nanoparticles for smart intravenous delivery of a practically-insoluble antineoplastic drug: development, preliminary safety evaluations and preclinical pharmacokinetic studies. Int J Pharm. 2015;493(12):439-450. doi:10.1016/j.ijpharm.2015.08.011

44. Reisch A, Runser A, Arntz Y, Mély Y, Klymchenko AS. Chargecontrolled nanoprecipitation as a modular approach to ultrasmall polymer nanocarriers: making bright and stable nanoparticles. ACS Nano. 2015;9(5):5104. doi:10.1021/acsnano.5b00214
45. Hoa LT, Chi NT, Nguyen LH, Chien DM. Preparation and characterization of nanoparticles containing ketoprofen and acrylic polymers prepared by emulsion solvent evaporation method. J Exp Nanosci. 2012;7(2):189-197. doi:10.1080/17458080.2010.515247

46. Al-Mahallawi AM, Abdelbary AA, Aburahma MH. Investigating the potential of employing bilosomes as a novel vesicular carrier for transdermal delivery of tenoxicam. Int J Pharm. 2015;485(1-2):329-340. doi:10.1016/j.ijpharm.2015.03.033

47. Khalil RM, Abdelbary GA, Basha M, Awad GEA, El-Hashemy HA. Enhancement of lomefloxacin $\mathrm{Hcl}$ ocular efficacy via niosomal encapsulation: in vitro characterization and in vivo evaluation. J Liposome Res. 2017;27(4):312-323. doi:10.1080/08982104.20 16.1191022

48. Kocbek P, Obermajer N, Cegnar M, Kos J, Kristl J. Targeting cancer cells using PLGA nanoparticles surface modified with monoclonal antibody. $J$ Control Rel. 2007;120(1-2):18-26. doi:10.1016/j. jconrel.2007.03.012

49. Kedmi R, Ben-Arie N, Peer D. The systemic toxicity of positively charged lipid nanoparticles and the role of Toll-like receptor 4 in immune activation. Biomaterials. 2010;31(26):6867-6875. doi:10.10 16/j.biomaterials.2010.05.027

50. Rosca ID, Watari F, Uo M. Microparticle formation and its mechanism in single and double emulsion solvent evaporation. J Control Rel. 2004;99(2):271-280. doi:10.1016/j.jconrel.2004.07.007

51. Motwani SK, Chopra S, Talegaonkar S, Kohli K, Ahmad FJ, Khar RK. Chitosan-sodium alginate nanoparticles as submicroscopic reservoirs for ocular delivery: formulation, optimisation and in vitro characterisation. Eur J Pharm Biopharm. 2008;68(3):513-525. doi:10.1016/j.ejpb.2007.09.009

52. Zhang L, Guo J, Peng X, Jin Y. Preparation and release behavior of carboxymethylated chitosan/alginate microspheres encapsulating bovine serum albumin. J Appl Polym Sci. 2004;92(2):878-882. doi:10.1002/app.13708

53. Mudgil M, Pawar PK. Preparation and in vitro/ex vivo evaluation of moxifloxacin-loaded PLGA nanosuspensions for ophthalmic application. Sci Pharm. 2013;81(2):591-606. doi:10.3797/scipharm. 1204-16

54. Gupta H, Aqil M, Khar RK, Ali A, Bhatnagar A, Mittal G. Sparfloxacin-loaded PLGA nanoparticles for sustained ocular drug delivery. Nanomedicine. 2010;6:324-333. doi:10.1016/j.nano.2009. 10.004

55. Ravi S, Peh KK, Darwis Y, Murthy BK, Singh TR, Mallikarjun C. Development and characterization of polymeric microspheres for controlled release protein loaded drug delivery system. Indian J Pharm Sci. 2008;70(3):303-309. doi:10.4103/0250-474X.42978

56. Katara R, Sachdeva S, Majumdar DK. Enhancement of ocular efficacy of aceclofenac using biodegradable PLGA nanoparticles: formulation and characterization. Drug Deliv Transl Res. 2017;7 (5):632-641. doi:10.1007/s13346-017-0416-1

57. Vega E, Gamisans F, García ML, Chauvet A, Lacoulonche F, Egea MA. PLGA nanospheres for the ocular delivery of flurbiprofen: drug release and interactions. J Pharm Sci. 2008;97(12):5306-5317. doi:10.1002/jps.21383

58. Malhotra S, Khare A, Grover K, Singh I, Pawar P. Design and evaluation of voriconazole eye drops for the treatment of fungal keratitis. J Pharm. 2014;2014:490595.

59. Schoenwald RD, Huang HS. Corneal penetration behaviour of b-blocking agents I: physicochemical factors. $J$ Pharm Sci. 1983;72:1266-1272. doi:10.1002/jps.2600721108

60. Sahoo S, Chakraborti C, Mishra S, Nanda U, Naik S. FTIR and XRD investigations of some fluoroquinolones. Int J Pharm Pharm Sci. 2011;3(3):165-170.

61. Fu J, Dong XX, Zeng ZP, Yin XB, Li FW, Ni J. Preparation and physicochemical characterization of T-OA PLGA microspheres. Chin $J$ Nat Med. 2017;15(12):912-916. doi:10.1016/S1875-5364(18)300 $07-4$ 
62. Yadav KS, Rajpurohit R, Sharma S. Glaucoma: current treatment and impact of advanced drug delivery systems. Life Sci. 2019;221:362-376. doi:10.1016/j.1fs.2019.02.029

63. Lynch CR, Kondiah PPD, Choonara YE, Du Toit LC, Ally N, Pillay V. Hydrogel biomaterials for application in ocular drug delivery. Front Bioeng Biotechnol. 2020;8:228. doi:10.3389/ fbioe. 2020.00228

64. Dolz M, Herraez M, González F, Sales OD. Flow behaviour of Carbopol- $940^{\circledR}$ hydrogels. The influence of concentration and agitation time. Pharmazie. 1998;53(2):126-130.

65. Joshi SC. Sol-Gel Behavior of Hydroxypropyl Methylcellulose (HPMC) in ionic media including drug release. Materials (Basel). 2011;4(10):1861-1905. doi:10.3390/ma4101861

66. Wang YY, Hong CT, Chiu WT, Fang JY. In vitro and in vivo evaluations of topically applied capsaicin and nonivamide from hydrogels. Int J Pharm. 2001;224:89-104. doi:10.1016/S0378-5173(01)00755-4

67. Hasan Sathali AA, Sangeetha T. Formulation and evaluation of ocular niosomal in situ gel of levofloxacin hemihydrate. J Pharmacy Res. 2011;4:4331-4337.
68. Abdel-Rashid RS, Helal DA, Omar MM, El Sisi AM. Nanogel loaded with surfactant based nanovesicles for enhanced ocular delivery of acetazolamide. Int $J$ Nanomedicine. 2019;14:2973-2983. doi:10.2147/IJN.S201891

69. Pandurangan DK, Bodagala P, Palanirajan VK, Govindaraj S. Formulation and evaluation of voriconazole ophthalmic solid lipid nanoparticles in situ gel. Int J Pharm Investig. 2016;6(1):56-62. doi:10.4103/2230-973X.176488

70. Panyam J, Labhasetwar V. Biodegradable nanoparticles for drug and gene delivery to cells and tissue. Adv Drug Del Rev. 2003;55 (3):329-347. doi:10.1016/S0169-409X(02)00228-4

71. Beisel KW, Hazlett LD, Berk RS. Dominant susceptibility effect on the murine corneal response to Pseudomonas aeruginosa. Proc Soc Exp Med. 1983;172:488-491. doi:10.3181/00379727-172-41592

72. Hume EB, Dajes JJ, Moreau JM, Sloop GD, Willcox MD, O'Callaghan RJ. Staphylococcus corneal virulence in a new topical model of infection. Investig Ophthalmol Vis Sci. 2001;42:2904-2908.

\section{Publish your work in this journal}

Drug Design, Development and Therapy is an international, peerreviewed open-access journal that spans the spectrum of drug design and development through to clinical applications. Clinical outcomes, patient safety, and programs for the development and effective, safe, and sustained use of medicines are a feature of the journal, which has also been accepted for indexing on PubMed Central. The manuscrip management system is completely online and includes a very quick and fair peer-review system, which is all easy to use. Visit http://www. dovepress.com/testimonials.php to read real quotes from published authors. 